\title{
Structural Biology of Calcitonin: From Aqueous Therapeutic Properties to Amyloid Aggregation
}

\author{
Kian Kamgar-Parsi, ${ }^{[\mathrm{a}]}$ James Tolchard, ${ }^{[\mathrm{b}]}$ Birgit Habenstein, ${ }^{[\mathrm{b}]}$ Antoine Loquet, ${ }^{[\mathrm{b}]}$ Akira Naito, ${ }^{[\mathrm{c}]}$ and \\ Ayyalusamy Ramamoorthy*[d]
}

Abstract: Under appropriate conditions, peptides and proteins can assemble from their native state into prefibrillar oligomers and then mature into fibrillar aggregates. This transition forms the molecular basis of several pathologies, often related to the deposition of these amyloid fibrils. Several hormone peptides involved in fundamental biological processes have the tendency to self-assemble into amyloid fibrils, resulting in a loss of their native functions, and more importantly, entailing devastating consequences, such as the formation of amyloid depositions. Calcitonin is a 32 amino-acid hormone peptide that can be considered a molecular paradigm for the central events associated with hormone misfolding. Calcitonin in its native form is involved in various physiological functions, including mediating calcium homeostasis and maintaining bone structure. It is the latter function that has motivated the use of calcitonin as an aqueous therapeutic agent for the treatment of bone-related pathologies such as osteoporosis and Paget's disease. Despite some success as a therapeutic, calcitonin's ability to control these diseases is limited by its aggregation along the canonical amyloid aggregation pathway, compromising its long-term stability as a therapeutic agent. A better understanding of the misfolding process would not only provide the structural basis to improve calcitonin's long-term stability and activity as a therapeutic, but also provide valuable insights into pathological aggregation of other amyloids. In this work, we review the physiological roles of calcitonin, its structure, and aggregation process, and consider the effects of calcitonin's structure on its role as a therapeutic.

Keywords: amino acids · amyloid protein · calcitonin · protein misfolding · self-assembly

\section{Introduction}

Calcitonin (CT) was first discovered in 1962 by Copp and colleagues. $^{[1]}$ By monitoring serum calcium levels in fasted and anesthetized dogs, it was found that, in concert with the previously identified parathyroid hormone (PTH), an additional hypocalcemic factor named calcitonin, was involved in the maintenance of blood calcium levels. These findings were reaffirmed a year later by Kumar et al. ${ }^{[2]}$ Despite initial experimentalists ascribing a parathyroidal origin for $\mathrm{CT}$, it was soon determined to be a thyroid hormone. ${ }^{[3]}$ The 32 amino-acid sequence of human calcitonin (hCT) was first determined in 1968, and in the following years, several other CT variants were discovered and sequenced, including the oft-studied bovine, porcine, and salmon CTs. ${ }^{[4-7]}$ All sequenced CTs are 32 residues in length and have an intramolecular disulfide bridge (Cys1-Cys7), but sequence homology varies significantly from species to species, with hCT differing from other CTs by as few as 2 residues (murine CT) or as many as 19 residues (ovine CT) ${ }^{[8,9]}$ Interestingly, the presence of the N-terminal disulfide bond is well conserved across all known calcitonin sequences, a feature commonly thought to reduce amyloidogenic aggregation. ${ }^{[10]} \mathrm{A}$ [a] K. Kamgar-Parsi

Applied Physics Program

University of Michigan

Ann Arbor MI 48109-1040 (USA)

[b] J. Tolchard, B. Habenstein, A. Loquet Institute of Chemistry and Biology of Membranes and Nanoobjects

CNRS

CBMN

UMR 5248

University of Bordeaux

33600 Pessac (France)

[c] A. Naito

Graduate School of Engineering

Yokohama National University

79-5 Tokiwadai

Hodogaya-ku

Yokohama 240-8501 (Japan)

[d] A. Ramamoorthy

Department of Chemistry and Biophysics Program

University of Michigan

930 North University Avenue

Ann Arbor MI 48109-1055 (USA)

Tel.: (+1) 734-647-6572

e-mail: ramamoor@umich.edu 
Kian Kamgar-Parsi was born in Silver Spring, Maryland, USA and received a bachelor's degree in physics from the University of Virginia in 2012. He is currently a graduate student in the Applied Physics Program at the University of Michigan and doing Ph.D. thesis study in the laboratory of Professor Ayyalusamy Ramamoorthy. His research interest centers on the structural changes involved in the aggregation of human calcitonin and how these structural changes influence aggregation kinetics and receptor interactions/ biological activity. chemistry from the University of East Anglia, UK, under the supervision of Dr. Tharin Blumenschein, where his studies focused on the characterization of pathogen effector proteins by the application of solution NMR. Currently, he works as a postdoctoral researcher investigating biological supramolecular assemblies in the team of Dr. Antoine Loquet at the Institut Européen de Chimie et Biologie, Pessac, France.
Dr. James Tolchard earned his Ph.D. in

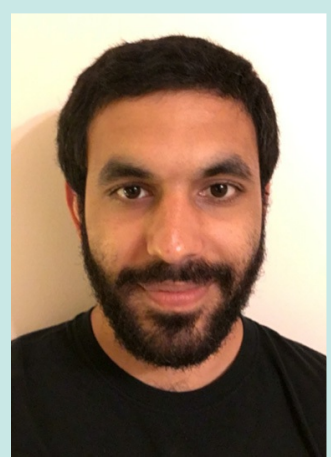

Dr. Antoine Loquet obtained his Ph.D. in biophysics with Dr. Anja Böckmann in 2009, and joined, as an EMBO postdoctoral fellow, the group of Prof. Adam Lange at MPI Göttingen from 2009 to 2013. During his time in Göttingen, he developed several approaches for determining atomic structures of supramolecular assemblies using solid-state NMR spectroscopy. In 2014, he established his laboratory at the IECB in Bordeaux and joined the CBMN as a CNRS researcher. He is

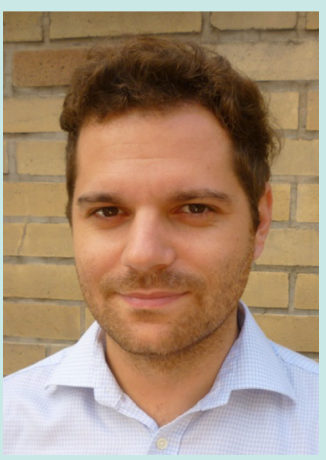
a recipient of an ERC Starting Grant to develop new NMR methods to study supramolecular assemblies.

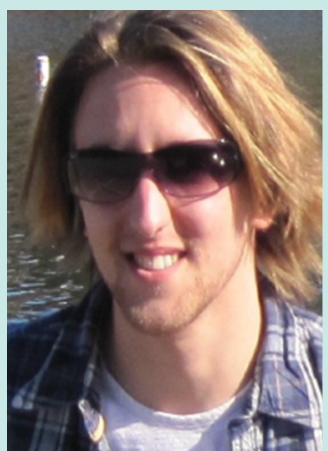

Dr. Akira Naito obtained his Ph.D. from the Department of Chemistry at Kyoto University, Japan, working with Professor Kazuyuki Akasaka on radiation damage of the disulfide radical as studied by ENDOR spectroscopy. He worked as a postdoctoral research fellow with Professor Charles A. McDowell at the University of British Columbia, Canada. Since 2001, he has been a Professor at Yokohama National University, Japan. His main research interests are developing photo and micro-

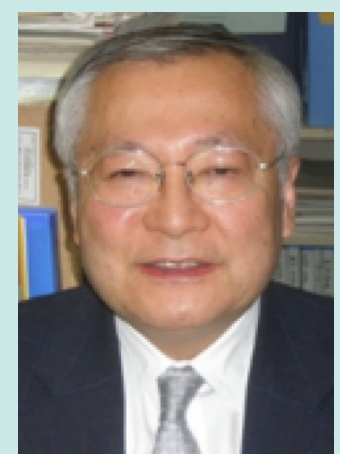
wave irradiation systems for solid-state NMR studies and applications of solid-state NMR methods to study biological systems such as amyloid peptides and membrane proteins.

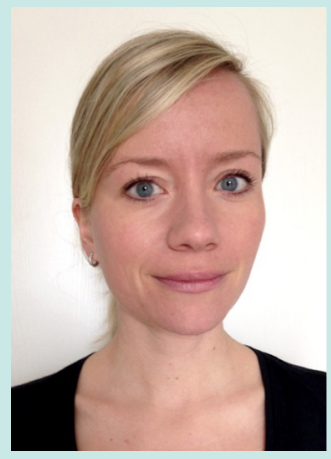

Dr. Birgit Habenstein received her Ph.D. in Lyon in 2011 under the supervision of Dr. Anja Böckmann, working on the structures of aggregating proteins by solid-state NMR. After a short postdoctoral stay at ETH Zurich with Professor Beat Meier, she joined, as an EMBO postdoctoral fellow, the team of Professor Adam Lange at the MaxPlanck-Institute in Göttingen to study the structure and assembly mechanism of several biological macromolecular complexes. In 2014, she obtained a CNRS senior researcher position to join the Institute of Chemistry and Biology of Membranes and Nanoobjects (CBMN) in Bordeaux. Her research focuses on the development and application of solidstate NMR to study aggregating proteins and membrane complexes. the Indian Institute of Technology
Dr. Ayyalusamy Ramamoorthy obtained his Ph.D. in Chemistry in 1990 from (Kanpur, India) working on the development of NQR spectroscopy. He subsequently moved to the Central Leather Research Institute (a national research laboratory in Madras/Chennai, India) as a Fellow Scientist to develop scalar coupling-based NMR methods. In 1992, he joined JEOL Ltd. (Tokyo, Japan) as a scientist in the laboratory of Professor Kuniaki Nagayama to de-

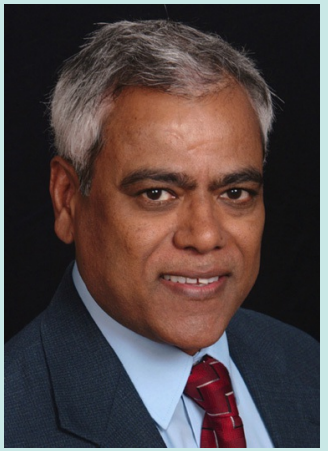
velop NMR techniques for studies on biological solids. He then joined Professor Stanley Opella's group (University of Pennsylvania, Philadelphia) in 1993 to further develop and apply solid-state NMR techniques for atomic-level resolution imaging of membrane proteins. In 1996, he joined the University of Michigan in Ann Arbor, where he currently holds a joint appointment as a professor in Biophysics and the Department of Chemistry. His main research interests are on the development and applications of NMR spectroscopy to study dynamic structures and function of membrane proteins and amyloid proteins. More details about his current research can be found at http://www.umich.edu/ ramslab. 


\section{hCT CGNLSTCMLGTYTQDFNKFHTFPQTAIGVGAP bCT CSSNLSTCVLSAYWKDLNNYHRFSGMGFGPETP PCT ĊSNLTCுVLSAYWRNLNNEHRFSGMGFGPETP SCT ĊSNLSTĆVLGKLSQELHKLQTYPRTNTGSGTP ECT ĆSNLSTĆVLGKLSOELHKLQTYPRTNDVSATP

$$
\text { *.*****:*. .: : : : : : : : * }
$$

Figure 1. Amino-acid sequence alignment of the commonly studied human, bovine, porcine, salmon, and eel calcitonin. All sequences contain a disulfide bridge between residues 1 and 7 , with 8 residues being conserved in all species $\left({ }^{*}\right)$, primarily at the $\mathrm{N}$-terminus. C-terminal amino-acid sequences vary significantly between different homologues. Evidence for the evolutionary divergence of different CT sequences can be seen in the relative conservation of CT between more recently diverged species. Eel and salmon CT (eCT and sCT) differ at only 3 locations (orange boxes), with bovine and porcine CT (bCT and pCT) also differing at 3 (blue boxes), whereas mammalian and fish CTs maintain less than $50 \%$ sequence homology. Colors describe residues of similar chemistry, asterisks denote fully conserved residues, and periods and colons indicate weak and strong conservation of amino-acid chemistry, respectively.

comparative alignment of several commonly studied calcitonin peptide sequences is shown in Figure 1.

Despite the sequence diversity among CT variants, all CTs have been shown to aggregate into amyloid fibrils when concentrated in aqueous conditions. ${ }^{[11-14]}$ The term "amyloid" references a specific type of supramolecular protein/peptide fibrillar architecture in which monomeric subunits are stacked along the fibril axis through intermolecular hydrogen bonds and electrostatic interactions between $\beta$-strands. ${ }^{[15,16]}$ Such an amyloid fold was structurally characterized for aggregated calcitonin peptides by the presence of $\beta$-rich conformation in hCT and the typical cross- $\beta$ structure in salmon CT by X-ray diffraction. ${ }^{[11,17]}$ This type of aggregation behavior and secondary structure change is observed in numerous peptides, and similar conformational diseases have been attributed to a wide variety of pathologies. ${ }^{[18]}$ A significant number of human amyloid peptides have been found to play a role in conferring pathological conditions to propagate diseases, including the extensively studied amyloid- $\beta$, human islet amyloid polypeptide (hIAPP), and $\alpha$-synuclein peptides, which are implicated in Alzheimer's disease, type 2 diabetes mellitus, and Parkinson's disease, respectively. ${ }^{[19-21]}$ Importantly, amyloids can reveal infectious properties, as has been demonstrated for the prion protein. ${ }^{[22]}$ Aggregation products and/or intermediates of these peptides/proteins have been shown to be toxic to various cell types or to drastically perturb the cellular functions. ${ }^{[19-21]}$

The aggregation process of these peptides is thought to be highly conserved, despite differences in primary sequences. ${ }^{[23,24]}$ For a detailed background and more details on the topic of amyloid aggregation, readers are referred to recent review articles in the literature. ${ }^{[16,25-29]}$ Briefly, unstructured monomers fold and combine to form small, intermediate aggregates (oligomers) during a period called the lag phase. Oligomers then gradually progress to generate larger protofibrillar species. Upon the formation of a critical nucleating species, amyloids can self-template and undergo rapid fiber growth occurring during the elongation phase. Eventually, an equilibrium is reached where maximal fiber content is achieved, known as the plateau phase. This description of amyloid aggregation is oversimplified; however, in reality, a complex amalgamation of off-pathway aggregates, species metastability, and heterogeneity serve to obfuscate the determination of the driving factors and intermediate species in the fibrillation and toxicity of amyloidogenic sequences (Figure 2). Additionally, aggregation is sensitive to a range of environmental factors, including buffer composition, salt, $\mathrm{pH}$, lipids, and metals, highlighting the difficulties inherent in generating an all-inclusive model of amyloid aggregation. ${ }^{[16,20]}$ Although amyloids were originally a species of interest in disease due to their propensity to form readily identified plaques, recent evidence has shifted focus away from mature aggregates and towards early oligomers as the putative pathological species in amyloid-related diseases. ${ }^{[16,20,21]}$ Significant efforts are ongoing to elucidate the mechanism(s) of toxicity and identify the toxic species in amyloid-related diseases.

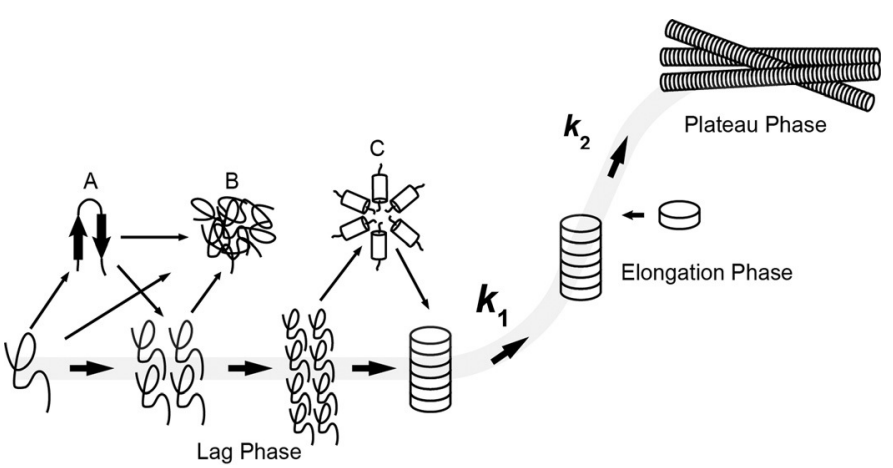

Figure 2. Pathway of typical amyloid aggregation pathway. The illustration presents a simplified overview of the process. Initially unstructured monomers aggregate into low molecular weight oligomers, which further aggregate into larger soluble oligomers during the lag phase. Upon formation of a critical nucleus/protofibrillar species, with a first time-determining rate constant, $k_{1}$, aggregation is self-templated and fibril elongation progresses rapidly through the addition of monomers or small oligomers in the elongation phase, with a second time-determining rate constant, $k_{2}$. Eventually, fiber formation rates equilibrate, with breakage rates and fiber content remaining constant; this is known as the plateau phase. Typically, a majority of initial monomers are sequestered in fibrils, but small aggregates persist even after fibrillation is complete. This picture is complicated by the number of off-pathway intermediates, which provide alternative pathways to fibrils $(A, C)$ and amorphous nonfibrillating aggregates (B). Secondary structural shifts in the monomer to $\beta$-sheet morphology $(A)$ and the formation of $\alpha$ helical peptide micelles (C) as critical species have also been proposed as steps in the amyloid cascade. 
The amyloidogenic aggregation of CT presents multiple practical challenges, both in normal bodily function and in therapeutic applications. While multiple variants of CT are known to be toxic and form membrane-permeabilizing oligomers in vitro, the roles that such behaviors play in vivo, if any, are not as well established as in other amyloids. ${ }^{[12,30-33]}$ To date, such behaviors have not been shown to have any significant roles in disease pathologies, though membrane interactions do hold relevance for both biological function and drug delivery. Studies of CT have revealed that it has skeletoprotective properties mediated through interactions with the calcitonin receptor (CTR), leading to its use as an osteoporosis therapeutic agent. ${ }^{[8,34]}$ Aggregation, however, remodels the monomeric subunits of CT and decreases their free concentration, consequently preventing the activation of CTRs. ${ }^{[35,36]}$ Efforts to combat this limited bioavailability have led to the replacement of hCT, as a therapeutic agent, with the slower-aggregating salmon CT (sCT). However, not only does SCT therapy cause immune response correlated complications, but hCT has also been found to be more potent than sCT under conditions where aggregation was controlled. ${ }^{[36-38]}$ Such considerations highlight why there is significant interest in manipulating the aggregation and structural remodeling of both human and salmon CT for therapeutic purposes. This review will examine the role of CT in normal bodily function, its use as a therapeutic agent, and focus on how recent results and advances in structural understandings of the aggregation pathway relate to CT biology and therapy.

\section{Production and Expression}

The first evidence of the existence of a hypocalcemic hormone was provided in 1962 by Copp et al. ${ }^{[1]}$ Using perfusion studies, it was found that decreases in serum calcium levels occurred too rapidly to be caused solely by changes in the expression of the previously known hypercalcemic PTH. This behavior was independently reproduced within the next year, and parathyroidectomy experiments initially suggested a parathyroidal origin for CT. ${ }^{[2]}$ Further experiments revealed the true source of CT (then called thyrocalcitonin to distinguish it from the thought-to-be separate calcitonin) to be the thyroid. ${ }^{[3]}$ Immunofluorescence experiments later determined CT to be produced solely and specifically in thyroid C-cells. ${ }^{[39]}$

The synthesis of CT is explained in detail in other reviews. ${ }^{[8,40]}$ Briefly, CT is coded for by the CALC I gene. Splicing of the gene transcript at exon 4 yields CT in thyroid C-cells, with alternative splicing producing calcitonin gene-related peptide (CGRP), a potent vasodilator, in neural cells. Both CT and CGRP are part of the calcitonin family, along with the structurally similar peptides hIAPP and adrenomedullin (AM), although unlike CGRP, hIAPP and AM are not alternative splice prod- ucts for CT. ${ }^{[41]}$ The 32-residue mature CT, with a disulfide linker between residues 1 and 7 , is subsequently released from thyroid C-cells following cleavage from a larger precursor protein. ${ }^{[8,40]}$

\section{Physiological Roles}

In the decades following CT's discovery, research has revealed it to have additional physiological roles. Most prominent among these is its role in skeletal protection, with hypercalcemia prevention and gastrointestinal interactions being secondary roles. However, some studies have shown minimal metabolic deviations upon removal of CT, leading to speculations regarding the significance of CT's physiological role. ${ }^{[42]}$ Here we discuss the roles of CT in skeletal protection and hypercalcemia prevention in the body, and consider the evidence for a role in gastrointestinal function.

\subsection{Skeletal Protection}

CT's most prominent physiological role is its interaction with the skeleton to moderate calcium homeostasis between blood and bone. CT serves to maintain bone mass primarily by inhibiting osteoclasts, a bone-associated cell type responsible for bone resorption. ${ }^{[4-45]}$ CT's interactions with osteoclasts are mediated by the CTR, a G protein-coupled receptor widely expressed on osteoclast membranes. ${ }^{[46]}$ Upon activation by CT, CTR causes a prompt loss in osteoclast ruffled border, a decreased ability of osteoclasts to acidify the bone-cell interstitial space, and decreased cellular motility. ${ }^{[47-49]}$ All of these effects inhibit osteoclast activity and lead to decreased bone resorption and increased skeletal mass. The related amyloidogenic peptides CGRP and hIAPP have demonstrated an ability to activate CTR-mediated inhibitory pathways in osteoclasts, although their interaction and subsequent inhibition is far weaker than the native substrate CT. ${ }^{[8]}$ The relative affinity of the CTR for different members of the calcitonin family is mediated by receptor activity modifying proteins (RAMPs), and as such, elucidating the behavior of RAMPs is the subject of significant scientific effort. ${ }^{[20,50,51]}$

In addition to interactions with the CTR, CT has other direct effects on osteoclast function. CT can alter the phosphorylation state of the focal adhesion proteins paxillin, FAK, and Pyk2. ${ }^{[52]}$ A CT-dependent disruption of the actin-ring structure associated with the sealing zone of osteoclasts has also been observed. ${ }^{[52,53]}$ Loss of focal adhesions leads to decreased efficiency in resorption and eventual decline in overall osteoclast numbers. ${ }^{[8]} \mathrm{CT}$ can also form calcium permeable pores in lipid bilayers, with some claiming that such ion flow could lead to osteoclast detachment from the bone matrix..$^{[32,33,54,55]}$ A role for calcium permeable CT pores in vivo requires further explo- 
ration, although calcium-dependent alterations of osteoclast function are known. ${ }^{[56,57]}$

Interactions between $\mathrm{CT}$ and two other bone-related cell types, osteoblasts and osteocytes, have also been observed, although such interactions are poorly understood. CT administration has the conflicting actions of both decreasing osteoblast function and enhancing osteoinduction. ${ }^{[58,59]}$ Such behaviors lead to decreased and increased skeletal strength, respectively, confounding efforts to determine the function of CT's interaction with osteoblasts. CT also protects both osteoblasts (no CTR expression) and osteocytes (CTR expression) from apoptosis, indicating an apparently CTR independent interaction with such cells. ${ }^{[60]}$ In addition, osteocyte production of sclerostin, a protein associated with a decrease in bone growth, is induced by sCT. ${ }^{[61]}$ These behaviors have led some to suggest that CT regulates bone turnover through both osteoclast and osteocyte interactions. ${ }^{[62]}$ Overall however, the role of CT in mediating osteoblast and osteocyte function remains unclear.

Several experiments where thyroidectomy had no significant effects on long-term bone mass have challenged the significance of CT's role amongst the range of boneregulating hormones. ${ }^{[42,63]}$ Experiments have also surprisingly shown increased bone mass in CT/CGRPKO mouse models, although such results are confounded by the action of CGRP ${ }^{[64]}$ Additionally, complications from cortical porosity and overactive bone resorption were observed at 12 months of age in the CT/CGRPKO mouse models. ${ }^{[65,66]}$ The release of osteoclasts from the inhibitory effects of CT after prolonged exposure and the degradation of hCT in human serum are also well-established phenomena, suggesting the effect of hCT on bone cells is short-lived. ${ }^{[67,68]}$ It is thus likely that CT's primary contribution to bone physiology is in short-term bone remodeling, or under conditions of significant calcium stress. ${ }^{[62,69]}$

In particular, CT is believed to play a significant role in calcium homeostasis during pregnancy and lactation, when maternal bone mass is under pressure from the calcium demands of the fetus or infant. CT stimulates the production of 1,25-dihydroxyvitamin $\mathrm{D}_{3} \quad(1,25 \mathrm{D}$, the active form of vitamin D), with elevated CT and 1,25D levels being associated with pregnancy and lactation, an apparently contradictory relationship, given the role of $1,25 \mathrm{D}$ in promoting resorption. ${ }^{[70-74]}$ However, CT has also shown the ability to increase intestinal absorption of calcium, and thus, it appears as if CT could serve the dual roles of increasing dietary calcium uptake, and promoting calcium transfer from bone to serum (through 1,25D) during lactation and pregnancy. ${ }^{[73]}$ This behavior would both protect the maternal skeleton and ensure sufficient calcium delivery to the fetus or infant. In a CT Null mouse model, the bone-mineral loss during lactation and the time for bone mass to return to normal were both increased by the lack of CT. ${ }^{[72]}$ Treatment with $\mathrm{SCT}$ rescued mineral loss and time to return to baseline. ${ }^{[72]}$ As such, a heightened physiological role for CT during pregnancy and lactation when the mother is under elevated demand for calcium, has been proposed..$^{[70,72,75]}$

\subsection{Hypercalcemia Prevention}

The potential for CT to counteract hypercalcemia has been implied since its discovery. ${ }^{[1,2]}$ While it exhibits no ability to directly sequester or transfer calcium, CT's hypocalcemic function can be explained by its inhibition of osteoclasts; a decrease in bone resorption and transfer of calcium from bone to blood would decrease serum calcium levels. CT in healthy individuals under normal calcium load has a minimal effect, but shows an ability to decrease serum calcium under conditions of endogenous addition of calcium or high bone turnover ${ }^{[69,72,76-78]}$ As such, it has been theorized that under normal conditions (no exogenous load, normal bone turnover), PTH governs the efflux of calcium from bone, with the influx being governed by the normal concentration gradient of calcium. Meanwhile, under conditions of acute hypercalcemia, CT can decrease the efflux of calcium from the skeleton, and thus, reduce serum calcium levels through its inhibition of osteoclastic activity. ${ }^{[79,80]}$ Such a view is in keeping with the observed behavior of osteoclastic release from CT inhibition. It is also supported by the upregulation of CT under conditions of high serum calcium, and the susceptibility (and subsequent CT-based rescue) of CT KO mice to hypercalcemia. ${ }^{[62,81]}$

\subsection{Gastrointestinal Function}

Some evidence suggests that CT could also play a role in gastrointestinal function. Intestinal absorption of calcium is increased by CT. ${ }^{[73]}$ Elcatonin, a synthetic eel calcitonin, has been shown to inhibit gastric and duodenal ulcers. ${ }^{[82]}$ Calcitonin receptor-like receptors have been localized to the human GI tract, and the peptide hormone gastrin, responsible for the secretion of gastric acid by parietal cells, significantly upregulates CT secretion. ${ }^{[7,83,84]}$ A similar effect has been found for several other gastrointestinal hormones, as well. ${ }^{[85]}$ The studies on GI hormone-induced CT secretion were, however, conducted with surgically isolated thyroid glands in situ with direct exposure of the thyroid to GI hormones; thus, it is unclear if the response has any biological significance. Overall, the interplay of CT and gastrointestinal function, if any, is still to be determined.

\section{Structural Studies on Calcitonin Aggregation}

CT's aggregation in solution is associated with noncovalent supramolecular assembly, leading to the precipitation of the peptide. At the macroscopic level, it results in sample gelation. Initial structural investigation of CT ag- 
gregation was primarily focused on these end-stage aggregates. ${ }^{[11,86-89]}$ Work by Arvinte and colleagues provided one of the first studies focused on the structure and kinetics of hCT fibrillation. ${ }^{[11]}$ Arvinte found higher peptide concentrations correlate with decreased lag time, a behavior that has been reproducibly observed for sCT, hCT, and other amyloids, both experimentally and in simulations. ${ }^{[88,90-92]}$ hCT also showed a propensity to form wellordered fibrillar aggregates, another common characteristic of other amyloidogenic sequences (Figure 3). ${ }^{[20,88,93-97]}$ TEM image of hCT indicates that short fibrils were observed at $\mathrm{pH} 7.5$, while long twisted fibrils were observed at $\mathrm{pH} 3.3$ (Figures $3 \mathrm{~B}$ and 3C). ${ }^{[98]}$ Interestingly, hCT exhibited both $\alpha$-helical and $\beta$-sheet structures in end-stage fibers, a deviation from the canonical view of amyloid peptides as maintaining a stable, exclusively $\beta$-sheet conformation content. Further studies have demonstrated that while $\alpha$-helical motifs may exist in the final equilibrium mixture, fibril morphology primarily relies on $\beta$-sheet conformation. ${ }^{[12,32,98-103]}$ Figure 4 shows typical NMR fingerprints, based on solution and solid-state NMR, of hCT in various forms (soluble and aggregated in mature fibrils).

Subsequent studies have yielded more detailed structural data on the amyloid fibrils of CT. Based on solidstate NMR spectroscopy, Naito and colleagues have revealed distinct behaviors for the central, N-terminal, and C-terminal regions of hCT. Early aggregates contain a central $\alpha$-helix, with a loop structure at the N-terminus and a random coil C-terminus (Figure 5, monomer). ${ }^{[98]}$ During fibril formation, the central helix converts to a $\beta$ strand, with the N-terminal loop remaining unchanged. ${ }^{[98]}$ The formation of this central $\beta$-strand also results in a shorter C-terminal in random coil conformation, with residues closer to the central region incorporating into the $\beta$-strand (Figure 5, fiber). ${ }^{[99]}$ The observation of a central $\alpha$-helix to $\beta$-strand conversion with a structured $\mathrm{N}$ terminus and unstructured C-terminus has been confirmed in follow up studies by both Naito and others. ${ }^{[86,87,93,103-106]}$ These secondary structural changes in CT fibrillation persist in both neutral and acidic environments, but $\mathrm{pH}$-dependent differences in the relative
A

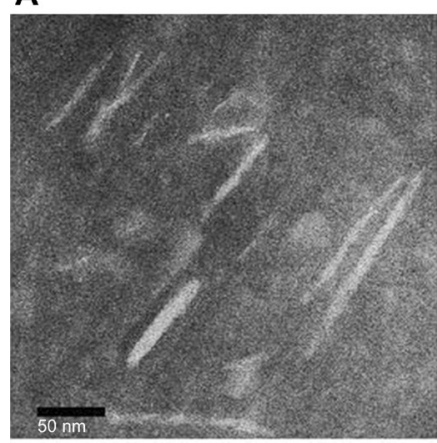

D

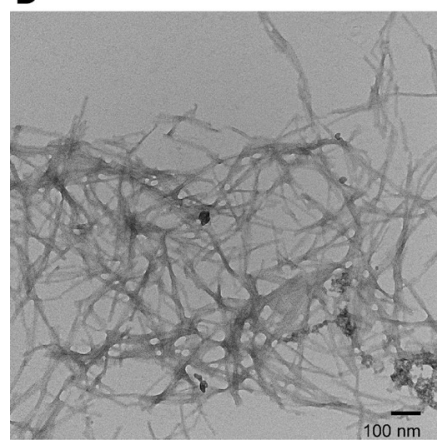

B

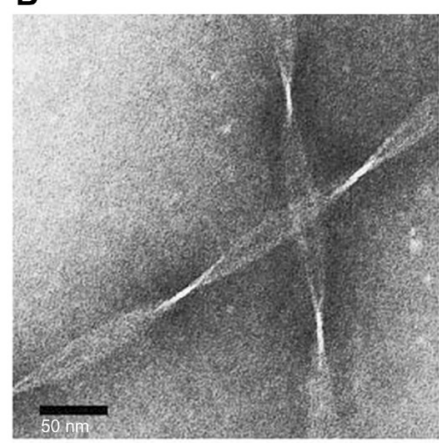

E

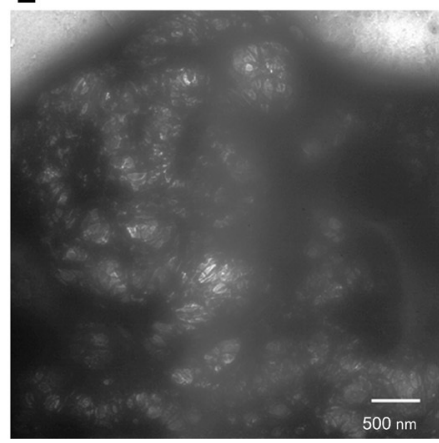

C

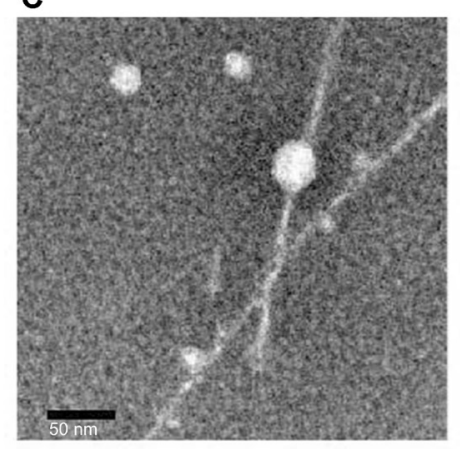

$\mathbf{F}$

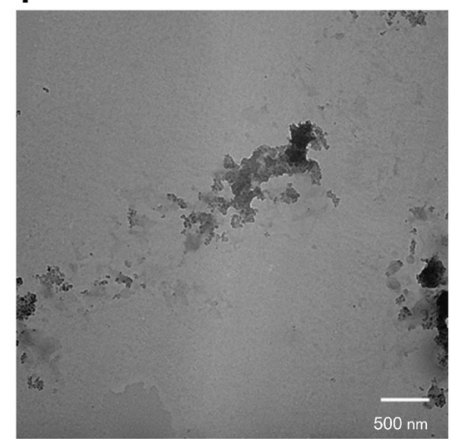

Figure 3. Transmission electron microscope images reveal polymorphic low-resolution structures of human calcitonin aggregates formed from solution samples prepared under different conditions. A) Short straight fibrils formed 90 min after dissolving $22.5 \mathrm{mg} \mathrm{ml}^{-1}$ of hCT in $20 \mathrm{mM}$ sodium phosphate buffer $(100 \mathrm{mM} \mathrm{NaCl}, \mathrm{pH} 7.5)$ at $20^{\circ} \mathrm{C}$. B) Long twisted ribbon type fibrils formed 45 days after dissolving $22.5 \mathrm{mg} \mathrm{ml}^{-1}$ of hCT in aqueous acetic acid $(15 \mathrm{mM}, \mathrm{pH} 3.3)$ at $20^{\circ} \mathrm{C}$. C) Spherical aggregates together with short fibrils formed 4 days after dissolving $40 \mathrm{mg} \mathrm{ml}^{-1}$ of hCT in HEPES solution $\left(20 \mathrm{mM}, \mathrm{pH}\right.$ 5.6) at $20^{\circ} \mathrm{C}$. D) Dense fibrils of hCT formed from $120 \mu \mathrm{M}$ initial monomer concentration at $25^{\circ} \mathrm{C}$ in $20 \mathrm{mM}$ sodium phosphate buffer (100,000 × magnification; unpublished results from the Ramamoorthy group). TEM images of $0.3 \mathrm{mM}$ samples of hCT after 36 hours of incubation at $\mathrm{pH} 7.4$ and $298 \mathrm{~K}$ : E) without; and F) with 3 molar equivalents of EGCG (epigallocatechin 3-gallate, a polyphenolic compound extracted from green tea). Absence of amyloid fibers in (F) suggests that EGCG effectively inhibits the aggregation of hCT, which has been further confirmed by NMR experiments; mechanistic details have been reported elsewhere by the Ramamoorthy group (Ref. [100]). TEM images A)-C) were adapted from Ref. [106], while E) and F) were adapted from Ref. [100]. 

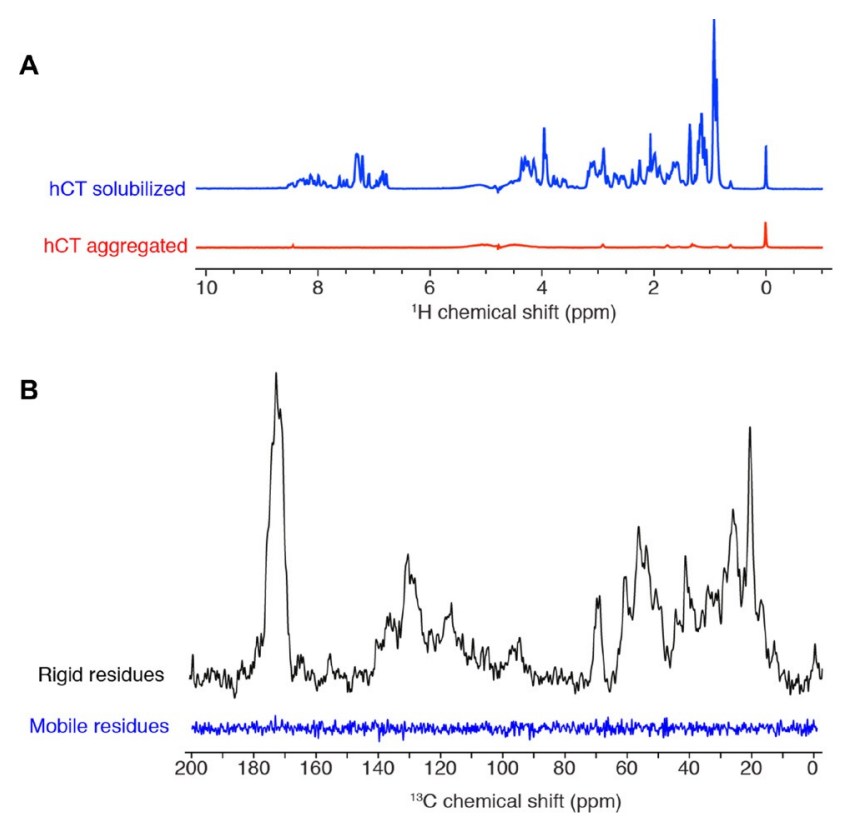

C

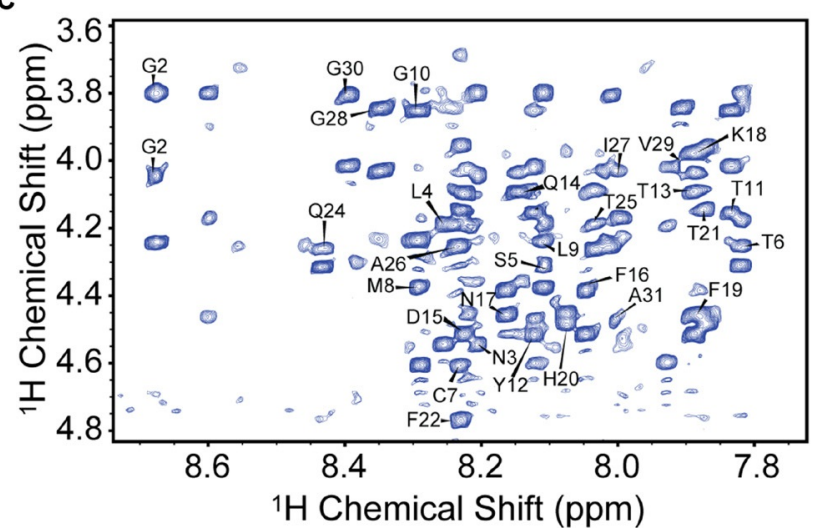

D
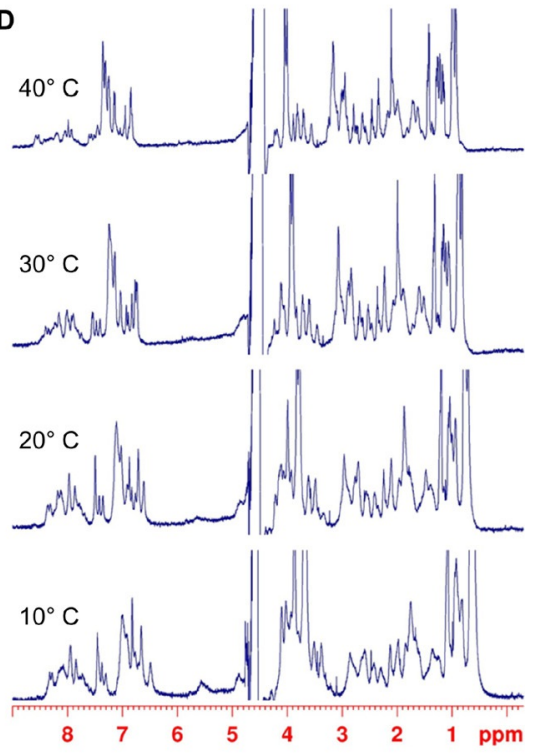

Figure 4. Solution and solid-state NMR spectra of hCT in various forms. A) Solution NMR spectra of hCT peptide, in the soluble form (in blue) and in its aggregated form (in red). Signal loss in the spectra of the aggregated form is due to the very slow molecular tumbling that exhibits prefibrillar and fibrillar nanoobjects. B) Solid-state NMR spectra of the hCT fibrils. Cross-polarization experiment (in black) probes the residues in a rigid conformation. The ${ }^{13} \mathrm{C}$ CPMAS spectrum shows relatively high resolution, indicative of a well-ordered core. INEPTbased polarization transfer from ${ }^{1} \mathrm{H}$ to ${ }^{13} \mathrm{C}$ (in blue) is used to probe mobile residues (faster than the microsecond timescale of motion). The absence of signals in the INEPT experiment indicates that all residues within the hCT peptide sequence adapt a rigid conformation. C) Twodimensional ${ }^{1} \mathrm{H} /{ }^{1} \mathrm{H}$ NOESY spectrum (the $\mathrm{H \alpha}-\mathrm{HN}$ region) of $1 \mathrm{mM} \mathrm{hCT}$ (in $2 \mathrm{mM}$ sodium phosphate buffer, $7 \% \mathrm{D}_{2} \mathrm{O}, 50 \mathrm{mM} \mathrm{NaCl}, \mathrm{pH}=2.9$ ) obtained at $900 \mathrm{MHz}$ proton resonance frequency with a $300 \mathrm{~ms}$ mixing time (spectrum adapted from Ref. [100]). NOEs were used to determine the structure of the peptide as reported elsewhere (Ref. [100]). D) Proton NMR spectra of hCT at pH 7.4 recorded at $600 \mathrm{MHz}$ shows the temperature-dependent spectral resolution; line broadening of amide-NH resonances and line narrowing of aliphatic resonances were observed as the sample temperature was increased from 10 to $40^{\circ} \mathrm{C}$, demonstrating the feasibility of probing the aggregation at atomiclevel resolution by NMR spectroscopy (unpublished results from the Ramamoorthy lab).

length and orientation of $\beta$-sheet motifs in the fibril do arise. ${ }^{[33,99,106]}$ It has been suggested that the charge states of the amino acids Asp15, Lys18, and His20 mediate this effect. ${ }^{[105]}$ It is likely, therefore, that electrostatic interactions play an important role in the structure and packing of CT fibers, as has also been observed for other amyloid peptide assemblies. ${ }^{[107,108]}$

The conformation of fibrils in acidic and neutral conditions was investigated by solid-state ${ }^{13} \mathrm{C}$ NMR spectroscopy using site-specific ${ }^{13} \mathrm{C}$ labeled hCT. ${ }^{[98]}$ The results indi- cate that the fibrils form an antiparallel $\beta$-sheet structure around Gly1- to Phe22 at pH 7.5 (Figure 5). ${ }^{[98]}$ On the other hand, the fibrils of hCT at $\mathrm{pH} 3.3$ were interpreted as forming a mixture of antiparallel and parallel $\beta$-sheets (Figure 5) ${ }^{[98]}$ Detailed $\beta$-strand packing in the hCT fibril grown at $\mathrm{pH} 7.5$ has been described by an accurately measured interatomic distance in the $\mathrm{D}_{15} \mathrm{FNKF}_{19}$ fragment, which is known as the minimum size of the peptide fragment to form a fibril. ${ }^{[99]}{ }^{13} \mathrm{C}$ and ${ }^{15} \mathrm{~N}$ nuclei in [1$\left.{ }^{13} \mathrm{C}\right]$ Phe16 and $\left[{ }^{15} \mathrm{~N}\right] \mathrm{Phe} 19$ of the $\mathrm{D}_{15} \mathrm{FNKF}_{19}$ fibril are lo- 

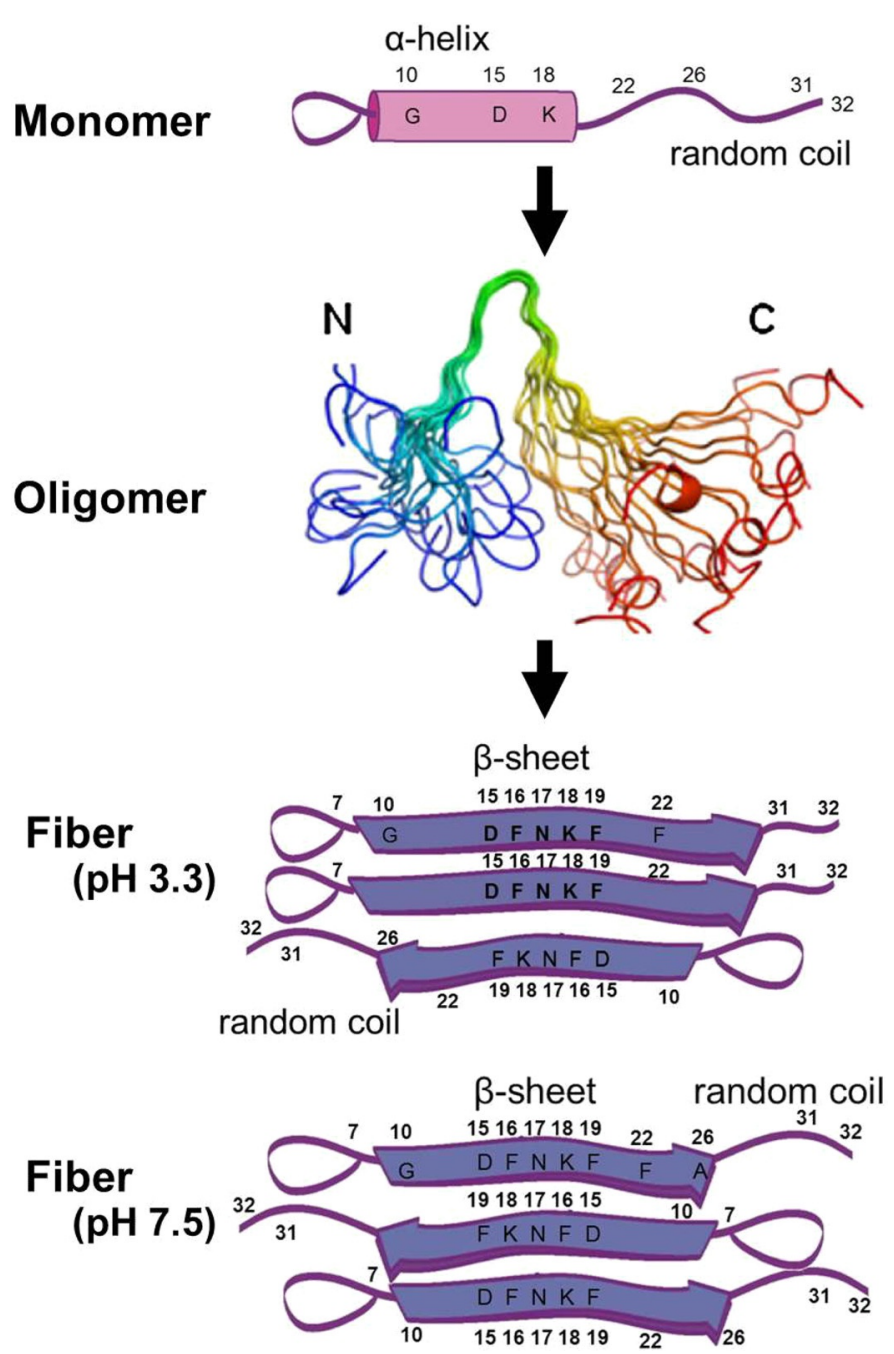

Figure 5. NMR structures of monomer, oligomer, and fibril stages of hCT. The monomer and fibril structural models are based on NMR experiments at $\mathrm{pH} 3.3$, and the oligomer structural model is based on NMR experiments at pH 3.3 and 7.5. The monomer exhibits an N-terminal loop, central $\alpha$-helix, and C-terminal random coil. During early stages of oligomerization, the helix transitions to a $\beta$ hairpin, with the $\mathrm{N}$ - and $\mathrm{C}$-termini remaining unchanged. This $\beta$ structure incorporates more C-terminal residues, with further aggregation, and eventually gives rise to a distinctive $\beta$-sheet morphology of amyloid fibrils. At low $\mathrm{pH}$, the charge states on residues 15,18 , and 20 allow for both parallel and antiparallel $\beta$-sheet stacking. At neutral $\mathrm{pH}$, residue 15 and 18 possess negative and positive charges, respectively; thus, fibrils show antiparallel $\beta$-stacking. The structures show one possible pathway from a monomeric to a fibril structure, although other intermediates along the way are likely.

cated one residue away from the known actual hydrogenbonding location in a head-to-tail antiparallel $\beta$-sheet structure. ${ }^{[99]}$ The phenyl rings of Phe16 and Phe19 residues are facing each other on the same side of the $\beta$ sheet, to be able to contact, via $\pi-\pi$ interactions, as illustrated in Figure 5. By assuming that the $\mathrm{D}_{15} \mathrm{FNKF}_{19}$ forms the core part of the hCT fibrils, the packing scheme of the hCT fibril at pH 7.5 is drawn as in Figure 5. ${ }^{[9]}$

Several other experiments have been carried out to probe the effect of electrostatic interactions on the aggregation kinetics of CT. At neutral $\mathrm{pH}$, hCT contains a negative charge at Asp15, and positive charges at Lys18 and the C-terminal amide. ${ }^{[105]}$ At acidic pH, His20 and Asp15 both become protonated, changing hCT's charge from +1 to $+3 .^{[105]}$ Increasing the peptide charge by decreasing the $\mathrm{pH}$ has been shown to slow aggregation in CT, yet substitution of the Asp15 with an Asn (increasing the net charge from +1 to +2 at neutral $\mathrm{pH}$ ) led to accelerated fibrillation kinetics. ${ }^{[100,105]}$ In contrast, a comparison, at neutral $\mathrm{pH}$, between hCT $(+1$ charge $)$ and the slower-aggregating sCT ( +3 charge) contradicts the behavior of the Asn-hCT mutant relative to the wild-type. ${ }^{[109]}$ The role of electrostatic interactions in CT-aggregation kinetics must therefore be more complicated than simple netcharge considerations. It is worth noting that all of hCT's charged residues occur in the central region (Figure 1), associated with rapid formation of $\alpha$-helices. Consequently, hydrophobic interactions that drive fibril formation likely confound efforts to elucidate the precise role of the charged residues in kinetics.

Naito's work has also implicated oligomeric intermediates with a spherical shape as important in seeding fibrils. ${ }^{[93]}$ Such intermediates were found to serve as nucleation sites for the growth and elongation of mature CT fibers, and appeared to be critical for fibril formation. While the structure of these fibers is similar to those previously observed, the biological relevance of the spherical intermediates is uncertain due to the known aggregationinhibiting properties of the fibrillation environment. ${ }^{[93]}$ Naito's studies have also demonstrated the importance of aromatic residue $\pi-\pi$ stacking interactions for maintaining fibril stability. ${ }^{[106]}$ This $\pi-\pi$ stacking is viewed as an important driving force during the self-assembly of amyloid fibrils, considering its energetic contribution to the intersubunit molecular interaction network and its impact into the directionality of the subunit stacking. ${ }^{[110]}$ In this regard, Metrangolo and coworkers have recently shown that iodination of the two phenalanine residues of hCT can amplify the fibril formation, leading to a 30-fold more efficient aggregation process. $^{[11]}$ The aromatic stacking is thought to be a major contributor to the kinetics difference between sCT and hCT, given that the involved central aromatics Tyr12, Phe16, and Phe19 in hCT all change to Leu in sCT (Figure 1). Such alterations would eliminate any stabilizing $\pi-\pi$ interactions, thus decreasing the stability of critical aggregation species and slowing fibrillation.

Similar to other central amyloidogenic peptide fragments, the central hCT pentapeptide fragment $\mathrm{D}_{15} \mathrm{FNKF}_{19}$ has been shown to be required for fibril formation. ${ }^{[103]}$ The central residues of $\mathrm{CT}$ are believed to form the fibrillar core of the amyloid fibrils, and the pen- 
tapeptide aggregates into fibers with a morphology similar to that formed by the full-length peptide. ${ }^{[98,101]}$ This fiber formation is noteworthy, due to the fairly hydrophilic nature of the pentapeptide, a challenge to the theory that amyloid aggregation is primarily driven by hydrophobic interactions, ${ }^{[12]}$ and rather, it suggests a combined mechanism of hydrophobic and hydrophilic interactions, as have been observed, for example, in polyQ disease-related peptides. ${ }^{[113]}$ Like the full-length peptide, it is possible that aromatic interactions play a major role in stabilizing the fibers, although experiments performed with other short peptide fragments from different amyloid peptides counter this claim. ${ }^{[103,114]}$ Such experiments instead suggest that aromatic interactions are not necessary for fibrillation, but simply alter fibril structure. Regardless, experiments have clearly demonstrated the $\mathrm{D}_{15} \mathrm{FNKF}_{19} \mathrm{hCT}$ fragment to be a key sequence in mediating fibril formation. A second amyloidogenic sequence of hCT towards the N-terminus, $\mathrm{T}_{6} \mathrm{CMLGT}_{11}$, has also been predicted, with an analog having been demonstrated to form amyloid fibrils, though the relevance of this fragment remains up for debate. ${ }^{[115]}$

A commonly observed feature of both sCT and hCT aggregation is the formation of $\alpha$-helical intermediates. ${ }^{[1,13,14,103,116-119]}$ Both the full-length CT and the $\mathrm{D}_{15} \mathrm{FNKF}_{19}$ pentapeptide have exhibited such structures, with $\alpha$-helical intermediates forming rapidly and being highly stable. ${ }^{[98,99,103]}$ The specifics of the progression from $\alpha$-helical intermediates toward the mature fibrillar structure are influenced by a number of factors, including $\mathrm{pH}$ conditions, the presence of lipids, buffer composition, and the concentrations and types of salts and metal ions. ${ }^{[13,33,93,96,98,99]}$ Despite this variability in fibrillation, $\alpha$ helical intermediates have been observed under a range of conditions, and play a significant role in the biological function of CT. ${ }^{[11,14,103,114,120-122]}$ Regarding the $\alpha$-helical intermediate, it is reported that dimerization of hCT stabilize the $\alpha$-helix under aqueous TFE solutions, leading to the long fibril formation. ${ }^{[123]}$ Within individual CT monomers, there are often multiple secondary structural motifs present that depend on the aggregation environment and could influence peptide behavior, but given the ubiquity of $\alpha$-helical intermediates, it seems generally accepted that they are a key species in the in vivo aggregation of CT. ${ }^{[98,99,104]}$

\subsection{Kinetic Analysis of Fibril Formation and Inhibition of Calcitonin}

Kinetic analyses of hCT were performed by using solidstate ${ }^{13} \mathrm{C}$ NMR to gain insight into the mechanism of fibril formation and inhibition. ${ }^{[93,98,99,105,106]}$ The increased ${ }^{13} \mathrm{C} \mathrm{CP} / \mathrm{MAS}$ NMR intensities suggest that the fibrillation can be explained by a two-step autocatalytic reaction mechanism, in which the first step is a homogeneous association to form a nucleus or intermediate, and the second step is a catalytic heterogeneous fibrillation to elongate the fibril. The rate constants for the first step $\left(k_{1}\right)$ and the second step $\left(k_{2}\right)$ were analyzed using the following equation: ${ }^{[98]}$

$f=\frac{\rho\{\exp [(1+\rho) k t]-1\}}{\{1+\rho \exp [(1+\rho) k t]\}}$

where $f$ represents the fraction of fibrils among the total population of peptides among the total population of peptide molecules: $\rho=k_{1} / k$; and $k=a k_{2}$, where the initial peptide concentration is given by $a$. The two-step autocatalytic reaction mechanism applied to kinetic analysis of hCT using Eq. (1) was found to be mathematically identical to the Finke-Watzky (W-T) two-step mechanism, which is also used for analysis of the kinetics of fibril formation. ${ }^{[124]}$ This kinetic analysis provide us with rate constant of the first step (fibril nucleation), $k_{1}$, which is responsible for the lag time of the fibrillation, and the rate constant of the second step (fibril elongation), which is responsible for the rate of fibril elongation.

In the kinetic analysis at $\mathrm{pH} 3.3$, the rate constant for the first step, $k_{1}$, values are three orders of magnitude smaller than that of the second step, $k_{2}$, values for the ${ }^{13} \mathrm{C}$ labelled hCT samples (Table 1). ${ }^{[98]}$ These results suggest that the first homogeneous nucleation process is much slower than the second heterogeneous fibrillation process. A fibrillation process derived by the kinetic analysis, as well as the conformational transition, is illustrated in Figure 2. Using this result, an $\alpha$-helical bundle (micelle) has to change its conformation from $\alpha$-helix to a $\beta$-sheet simultaneously in the first nucleation process, while one $\alpha$-helix can be converted to a $\beta$-sheet in the second heterogeneous fibrillation process. These findings, based on ${ }^{13} \mathrm{C}$ NMR experiments, imply that it is sufficient to consider a two-step reaction for the fibrillation kinetics. The time course of the fibril formation at $\mathrm{pH} 7.5$ was also examined by $\mathrm{CD}$ measurement, because it is not possible to determine the fast fibrillation rate at $\mathrm{pH} 7.5$ by ${ }^{13} \mathrm{C} \mathrm{NMR}$ measurements. ${ }^{[98]}$ The monomeric component assigned to the random coil $(205 \mathrm{~nm})$ in the CD spectra decreased gradually as a result of fibril formation in much lower concentrations than those for NMR measurements. Consequently, the kinetic parameters are observed in the same way, and it is found that the fibril formation after the nucleation at $\mathrm{pH} 7.5$ occurred much faster than that at $\mathrm{pH} 3.3$, because the value at 7.5 was three orders of magnitude larger than that at $\mathrm{pH} 3.3$, because $k_{1}$ values were not different among them. It is revealed that $k_{1}$ values are not correlated with $\mathrm{pH}$ and concentration from the experimental observations. ${ }^{[98,105]}$

The mutation from the aromatic side-chain (Y12, F16, F19) in hCT to Leu residues (TL-, F16L-, and F19L-hCT) resulted in a significantly slower elongation rate of fibril formation, $k_{2}$, specifically at neutral $\mathrm{pH}$ conditions than 
Table 1. $\mathrm{pH}$-dependent rate constants, $k_{1}$ and $k_{2}$, in a two-step autocatalytic reaction mechanism for $\mathrm{hCT}$ fibril formation in solution.

\begin{tabular}{lllll}
\hline Sample & Condition & $k_{1}\left(\mathrm{~s}^{-1}\right)$ & $k_{2}\left(\mathrm{~s}^{-} 1 \mathrm{M}^{-1}\right)$ & \\
\hline Wt-hCT & $\mathrm{pH} \mathrm{7.5}$ & $2.8 \times 10^{-6}$ & 2.3 & Ref. \\
Wt-hCT & $\mathrm{pH} \mathrm{3.3}$ & $3.3 \times 10^{-6}$ & $2.4 \times 10^{-3}$ & {$[98]$} \\
D75N-hCT & $\mathrm{pH} \mathrm{7.2}$ & $1.6 \times 10^{-5}$ & 5.5 & {$[98]$} \\
D75N-hCT & $\mathrm{pH} 2.9$ & $6.2 \times 10^{-7}$ & $5.4 \times 10^{-3}$ & {$[105]$} \\
F16L-hCT & $\mathrm{pH} \mathrm{7.5}$ & $1.9 \times 10^{-7}$ & $4.8 \times 10^{-2}$ & {$[105]$} \\
F16L-hCT & $\mathrm{pH} \mathrm{2.6-3.0}$ & $1.8 \times 10^{-6}$ & $6.2 \times 10^{-3}$ & {$[106]$} \\
F19L-hCT & $\mathrm{pH} \mathrm{7.5}$ & $7.4 \times 10^{-9}$ & $2.9 \times 10^{-2}$ & {$[106]$} \\
F19L-hCT & $\mathrm{pH} \mathrm{2.6-3.0}$ & $1.3 \times 10^{-6}$ & $1.6 \times 10^{-2}$ & {$[106]$} \\
TL-hCT & $\mathrm{pH} \mathrm{7.5}$ & $3.4 \times 10^{-6}$ & $3.0 \times 10^{-3}$ & {$[106]$} \\
TL-hCT & $\mathrm{pH} 2.6-3.0$ & $5.4 \times 10^{-6}$ & $4.8 \times 10^{-3}$ & {$[106]$} \\
Wt-hCT (HEPES) & $\mathrm{pH} \mathrm{5.6}$ & $3.0 \times 10^{-10}$ & $2.0 \times 10^{-3}$ & {$[106]$} \\
\hline
\end{tabular}

[a] F16L, F19L, Y22L-hCT.

that of hCT. In the case of hCT, since the aromatic sidechains are Tyr12, Phe16, Phe19, and Phe22, the side-chain of Phe16 and Phe19 could engage in $\pi-\pi$ stacking in an antiparallel $\beta$-sheet. If the Phe residue plays an essential role in determining the molecular packing, the sidechains of Asp15 and Lys18 changing at neutral pH might help the intermolecular packing, because the residues are located very closely to Phe16 and Phe19. In the hCT mutant, lack of Phe16 and Phe19 interaction causes significant instability, as compared with the Wt-hCT fibril. This instability of the $\beta$-sheet causes the reduction of $k_{2}$ values (Table 1). ${ }^{[06]}$

The two-step autocatalytic reaction mechanism was applied to the analysis of the kinetics of hCT fibrillation in HEPES solution. ${ }^{[93]}$ The nucleation rate, $k_{1}$, in HEPES solution at $\mathrm{pH} 5.6$ was $2.98 \times 10^{-10} \mathrm{~s}^{-1}$, which was significantly lower than the rate in neutral solution $\left(k_{1}=2.79 \times\right.$ $\left.10^{-6} \mathrm{~s}^{-1}\right)$. The fibril-elongation rate, $k_{2}$, in HEPES solution at $\mathrm{pH} 5.6$ was $1.98 \times 10^{-3} \mathrm{~s}^{-1} \mathrm{M}^{-1}$ (Table 1 ). This result indicates that HEPES shows a much slower fibril-nucleation rate than the fibril-elongation rate. Asp15 (negatively charged) and Lys18 (positively charged) in hCT may have strong electrostatic interaction with amino (positively charged) and sulfonyl (negatively charged) groups in HEPES, respectively. Because of the amphiphilic nature of the $\mathrm{N}$-terminus, $\alpha$-helices associate with each other to form micelle-like spherical aggregates. These aggregates may be further stabilized by the interaction with HEPES at the surface in the interfacial region of spherical aggregates, leading to the slow rate of nucleation. Because the spherical intermediates are stabilized, it is possible to observe the spherical intermediate of hCT in HEPES solution by TEM (Figure 3C). ${ }^{[93]}$

\subsection{Membrane Interactions}

CT-membrane interactions have been studied extensively, both for the possibility of physiological relevance via pore formation, and for the increasing prevalence of lipid-based delivery options for therapies. ${ }^{[32,33,104,125-127]}$ As with other amyloid peptides, CT preferentially interacts with membranes containing anionic lipids, cholesterol, and GM1 ganglioside. ${ }^{[16,20,24,32,114,128,129]} \alpha$-helical motifs in CT are often detected in membrane environments, and research suggests that helical intermediates are stabilized by lipids, and that such intermediates encourage stronger interactions with membranes, enhancing aggregation. ${ }^{[33,130,131]}$ Experiments have also shown that cholesterol-rich lipid environments enhance $\beta$-sheet content, likely the result of lipid-mediated fibril formation. ${ }^{[132]}$ It seems that the initial species to interact with the membranes is $\alpha$-helical and that further aggregation in the membrane environment allows fibrillation to continue, eventually leading to $\beta$-sheet containing fibrils. It would therefore appear that $\alpha$-helical intermediates of CT are key for aggregation in both solution and membrane environments.

Additional work by Diociaiuti and colleagues has implicated lipid rafts as having a crucial role in mediating the pore formation of sCT oligomers, a behavior observed in other amyloids, as well. ${ }^{[129]}$ The enrichment in lipid rafts for cholesterol and GM1 suggests that these membrane components could play a role in mediating interactions, although a role for lipid raft-induced changes in membrane thickness or curvature has not been ruled out. ${ }^{[54]}$ Work by Sheynis and Jelinek showed an interesting behavior of CT to form fibril mats on membrane surfaces without insertion, in contrast with the amyloid$\beta$ whose lipid-induced fibrillation is associated with a complete membrane rupture. ${ }^{[97,128]}$ While the possible implications of lipid raft-mediated CT membrane interactions on pore formation are clear, the potential physiological roles for fibril mats, if any, remain unknown.

\subsection{Prefibrillar Oligomers}

Compared with other amyloids, there are relatively few studies focused on prefibrillar aggregates of CT. However, some trends have emerged from the literature. Both annular and linear protofibrillar aggregates of SCT have been observed and characterized by Diociauti and colleagues, using both TEM and circular dichroism measurements, showing them to be primarily rich in random coil 
and $\beta$-strand conformation, respectively. ${ }^{[12]}$ Despite the prevalence of these larger species of prefibrillar aggregates, smaller $(<20$ mer $)$ prefibrillar oligomers are the species putatively responsible for membrane permeabilization, exhibiting enhanced pore-formation activity. ${ }^{[12,54,100,109]}$ As with several other amyloid peptides, pore formation by $\mathrm{CT}$ oligomers leads to calcium leakage and loss of cell homeostasis, with this behavior being modified by both membrane composition and membrane structure. ${ }^{[32,33,54,55,89]}$ As mentioned previously, the biological relevance of such pore formation remains unknown. Of greater interest therapeutically is the fact that oligomers are thought to form critical intermediates for further aggregation of CT, making them a potential target for inhibition. ${ }^{\text {[93] }}$

There is a relative lack of structural knowledge on early oligomers in CT, compared with mature fibrils, beyond the consistent formation of a central $\alpha$-helix. Among the few characterizations of prefibrillar oligomers, an NMR-based structural model for hCT at acidic $\mathrm{pH}$, obtained by Huang and colleagues, indicates the formation of a $\beta$-hairpin structure in the central region, with an N-terminal loop and unstructured C-terminus. ${ }^{[100]}$ This structure is believed to be stabilized by aromatic residue $\pi-\pi$ interactions, and is consistent with the structures of Naito and colleagues, representing a potential intermediate in the transition from $\alpha$-helix to $\beta$-strand (Figure 5). However, intermediates containing random coil, $\alpha$-helix, and $\beta$-hairpin secondary structures have all been detected in CT oligomers, highlighting the heterogeneity and metastability of early aggregation products and the significant effect of environmental conditions. ${ }^{[12,100,104]}$ It is likely that the oligomeric intermediate of Huang and colleagues represents only one of a number of oligomeric intermediates formed between monomers and fibers. Amongst these intermediates, micelle-like oligomers have been implicated in the aggregation of several other amyloid peptides, and unpublished results suggest they may play a role in CT aggregation, as well. ${ }^{[0,133]}$ Efforts to probe the role of these possible intermediates are ongoing.

\section{Role of Calcitonin Structure and Aggregation in Biological Function and Therapy}

For many of the more widely studied amyloid peptides, such as amyloid- $\beta$ and hIAPP, the biological relevance of their aggregation derives directly from their influence on disease states. Specifically, the formation of cytotoxic oligomers and subsequent cell death is believed to be directly responsible for several types of dementia and other pathologies. While CT has reproducibly demonstrated the ability to form toxic species in model membrane systems and cultured cells, to date, the aggregation of CT in organisms has neither been shown to directly cause significant toxicity, nor to drive any disease pathology. ${ }^{[13,30]}$ The relevance of in vivo CT aggregation is instead primarily related to its roles in mitigating peptide-receptor interactions and limiting therapeutic efficiency through alterations of peptide structure and bioavailability.

\subsection{The Role of CT Structure on Biological Function}

Research points to $\alpha$-helical intermediates as being key for several essential behaviors of CT. $\alpha$-helical content is directly proportional to biological activity in several CT variants. ${ }^{[14]}$ In the therapeutically relevant $\mathrm{SCT}$, the formation of an $\alpha$-helix between residues 9 and 19 may be a key structural determinant in mediating CT-CTR interactions, with this helix location being consistent with previously determined structures of sCT. ${ }^{[55,118,119,134]}$ Interestingly, sCT mutants with excess helical content have exhibited decreased receptor binding affinity and hypocalcemic activity, indicating that the specific length of $\alpha$-helical domains influences biological function. ${ }^{[121,135]}$ The central $\alpha$ helix of hCT is significantly shorter than that of the sCT, and hCT has shown superior hypocalcemic activity under aggregation-inhibiting conditions. ${ }^{[36,134]}$ A potential explanation is that the shorter $\alpha$-helix of hCT is superior for receptor activation, but either the shorter $\alpha$-helix or other structural elements of hCT also render it more prone to further aggregate. Thus, it appears that there is a balancing act between monomer potency and stability for CT and its various isoforms.

Mechanistically, several ideas exist as to how specific secondary structural elements mediate CT-receptor interactions. The N-terminal tail of CT, the most conserved section of CT across all species (Figure 1), is believed to be critical for receptor activation, binding to the CTR and initiating its cAMP cascade. ${ }^{[50,55,116,121,122]}$ This N-terminal region forms a loop structure in hCT as mentioned, with this structural motif persisting in a range of solution conditions. Studies with hCT peptide fragment 9-32 have shown helix formation upon interactions with membranes, but have failed to demonstrate spontaneous membrane insertion without the N-terminal residues, indicating the potential dual role of such residues in both receptor activation and membrane insertion. ${ }^{[116]}$ As mentioned previously, the presence and structure of $\alpha$-helical motifs is also correlated with CT biological activity. Additionally, NMR experiments, along with the well-established ability of $\alpha$-helical CT to insert into the membrane, led Rawat and Kumar to propose that CT could interact with intermembrane receptor domains, although such a behavior would be rare amongst GPCRs. ${ }^{[104]}$ Overall, it is likely that both the central $\alpha$-helix and $\mathrm{N}$-terminal loop contribute to receptor activation.

A role for the C-terminal residues of $\mathrm{CT}$ in receptor binding and activation is less clear, although recent results suggest they are involved. Johansson and colleagues recently managed to crystalize $\mathrm{sCT}$ C-terminal peptide fragments bound to the ectodomain of the CTR. ${ }^{[136]}$ 
Using X-ray crystallography, it was found that a type II $\beta$ turn formed involving residues $28-31$ of sCT, with this turn being complementary to a loop consisting of residues 121-128 of the CTR. Hydrogen bonding between sCT and CTR was also mapped, with stacking between Pro32 of sCT and Trp79 of CTR, in particular, being implicated as a potential key interaction. Working with similarly truncated sCT and CTR models, Lee and colleagues likewise found C-terminal residues of $\mathrm{SCT}$ to form a turn and hydrogen bond with CTR residues. ${ }^{[50]}$ This work also implicated the stacking of Pro32 of sCT and Trp79 of CTR as a potentially key mediator of CT-CTR interactions, with a P32Y mutation in the sCT sequence significantly weakening binding to the CTR ectodomain. These results suggest that $\mathrm{C}$-terminal residues of CT could mediate interactions with the CTR.

Despite these recent discoveries, evidence exists that the C-terminus is not relevant for receptor interactions. The unstructured nature of the CT C-terminus, along with the lack of sequence conservation between species, suggests a minimal role in receptor activation. ${ }^{[55,86,87]} \mathrm{C}$ terminally mutated hCT peptides have shown no decrease in receptor binding or hypocalcemic effect, calling into question whether or not the binding exhibited by Johansson and Lee was sufficient for receptor activation. ${ }^{[121,137]}$ The use of truncated versions of sCT and CTR by Johansson and Lee, along with the lack of a membrane environment and nonevaluation of activation also presents questions as to the biological relevance of the interactions observed. ${ }^{[50,136]}$ Clearly, further experiments involving CT and CTRs are necessary to clarify the structure-function relationship in CT-mediated CTR activation.

\subsection{The Role of CT Aggregation in Therapeutic Applications}

Owing to its roles in mediating bone reformatting, CT has been used as a therapeutic in the treatment of the bone-related diseases osteoporosis and Paget's disease to decrease the loss of bone mass and the rate of bone reformatting, respectively. ${ }^{[34,138]}$ For details on the history of CT therapy, the reader is directed to a review by Henrikson et al. ${ }^{[139]}$ Typically, sCT has been the most commonly used isoform in therapeutic applications, owing to its slower aggregation rate, relative to $\mathrm{hCT}$, which increases the amount of soluble, bioavailable monomeric peptide. ${ }^{[35]}$ Despite its slower aggregation relative to hCT, sCT still aggregates, making it an improvement over hCT, but still an inefficient therapeutic. ${ }^{[11,106]} \mathrm{SCT}$ therapy is also associated with several side effects, including anorexia and vomiting, and has been shown to cause antibody formation and immune response. ${ }^{[37,38,140,141]}$ Such reactions further decrease the efficacy of sCT treatments. ${ }^{[142]} \mathrm{hCT}$ is not associated with an immune response, and therefore modifications to the hCT peptide to increase its bioavailability are an attractive alternative to current $\mathrm{SCT}$ therapies. ${ }^{[143]}$
Promising targets for improving hCT bioavailability are the central aromatic residues Tyr12, Phe16, and Phe19. Mutation studies replacing these central aromatic residues in hCT with Leu (thus increasing homology with $\mathrm{sCT}$ ) led to a decrease in the aggregation rate by $2-3$ orders of magnitude. ${ }^{[106]}$ The more conservative F19Y mutation had no effect on aggregation kinetics, indicating that the effect of the central aromatics was general and not specific to any one amino acid. ${ }^{[106]}$ These findings were supported by MD simulations. ${ }^{[106]}$ Crucially, such mutants of hCT maintain their biological function, likely through stabilization of helical motifs, with both hypocalcemic potency and duration being enhanced over wt-hCT in rat models. ${ }^{[144,145]}$ Given their significant homology to wt-hCT, it is also unlikely that such mutants would elicit immune response. Additionally, polyphenols, a popular class of small molecule inhibitors of amyloid aggregation, have proven effective in modulating the aggregation of CT. ${ }^{[100,146]}$ It is believed that such inhibitors operate through interference with $\pi-\pi$ interactions, validating this region as a potential site for pharmaceutical optimization and further strengthening arguments for a strong role for aromatic residues in CT aggregation and remodeling. ${ }^{[100]}$

Other modifications to hCT could also be made to decrease its aggregation propensity. The oxidation state of methionine at residue 8 in hCT has been shown to alter its aggregation rate in a $\mathrm{pH}$-independent manner. ${ }^{[147]}$ The absence of methionine in $\mathrm{sCT}$ suggests that a mutation at residue 8 of hCT could present another avenue for kinetics modification. Investigation by Fowler and colleagues found that five amino-acid substitutions (T11R, N17R, N24R, I27T, V29S) in hCT could slow its aggregation to the rate of $\mathrm{sCT}$, mainly through increasing $\alpha$-helix stability and decreasing peptide hydrophobicity. ${ }^{[121]}$ Additionally, more of this $\alpha$-helix promoting hCT mutant remained soluble once aggregation was completed, compared with wild-type, and the peptide modifications did not adversely affect receptor binding or the hypocalcemic effect. Another study by Andreotti and colleagues found that the mutation of five central and C-terminally located residues in hCT to their sCT equivalents (Y12L, N17H, A26N, I27T, A31T) both decreased the rate of aggregation and maintained structure and biological function. ${ }^{[137]}$ This modified hCT also had the ability to inhibit the aggregation of wild-type hCT when the two were coincubated. ${ }^{[137]}$ As with the mutations to the aromatic residues of hCT, the mutants of Fowler and Andreotti would also be expected to decrease the immune responses endemic of $\mathrm{sCT}$ therapy, given the rarity of antibody formation against hCT. ${ }^{[143]}$ The decreased immune response and subsequently decreased secondary resistance would further enhance the effectiveness of CT therapies. Such mutated hCT variants thus present another potential alternative to $\mathrm{SCT}$ therapies.

CTs interactions with lipid vesicles are also of significant interest due to the increasing prevalence of lipid- 
based delivery systems for biomolecular therapeutics. $^{[125,126,147,148]}$ Significant efforts have been made to modify CTs to enhance their permeability through epithelial layers to increase their effective bioavailability. It appears that $\alpha$-helical intermediates would be beneficial to that end, due to their enhanced membrane insertion abilities. The formation of $\alpha$-helices by CT is enhanced by anionic lipids and cholesterol within the bilayer, and while such CT structures are believed to be key for receptor interactions, $\alpha$-helical intermediates in the presence of lipid bilayers also have been shown to drive fibril formation. A universal answer as to whether or not $\alpha$-helices are desirable during drug application thus appears unlikely, and would depend heavily on other factors (other peptide modifications, mode of intake, dosage, etc.). The choice of lipids in a lipid-based delivery system, particularly the head-group charge, could prove to be an attractive and simple means of controlling aggregation during peptide delivery.

Despite the prevalence of helical intermediates, mature CT fibrils are found to contain primarily a $\beta$-sheet secondary structure. ${ }^{[12,32,98-103]}$ The progression of CT monomers to $\beta$-sheet fibrils via $\alpha$-helical intermediates would seem to mark such intermediates as a target species for fibrillation inhibition, but any attempts at modifying CT fibrillation must be performed with receptor interactions in mind. As we have discussed, significant evidence exists showing $\alpha$-helical intermediates to be key in mediating peptide-receptor interactions. Therefore, direct inhibition of helix formation would likely render CT ineffective as a therapeutic. However, prevention of a further progression to $\beta$-strand morphologies and fiber formation would leave biological activity intact. The stabilization of helical intermediates has already been demonstrated in other systems to prevent fibrillation in vivo and enhance CT pore formation, indicating an increased bioavailability. ${ }^{[89,145,149-151}$ Furthermore, decreased fibrillation and improved bioavailability and function are observed in $\alpha$ helix promoting hCT mutants, and residue-specific data exists on the effect that modifying helix length and location has on peptide function. ${ }^{[121,134,135]}$ Therefore, stabilizing the formation of $\alpha$-helical intermediates of CT appears to be one of the simplest, and least invasive, methods by which to enhance the bioavailability and efficacy of CT as a therapeutic.

\section{Current Calcitonin Therapy}

The availability of more effective osteoporosis drugs, such as bisphosphonates, has led to CT therapy primarily being used for the short-term mediation of acute pain from osteoporosis, although combination treatments involving CT have shown efficacy in mediating both hypercalcemia and bone quality under circumstances in which other treatments were ineffective. ${ }^{[152-155]}$ In particular, CT has seen significant use as a therapeutic for the treatment of postmenopausal osteoporosis. ${ }^{[35,152,156,157]}$ Efforts to improve CT efficacy in recent years have primarily focused on peptide modifications and alternative delivery methods.

\subsection{Peptide Alterations}

In addition to preventing aggregation, therapeutic forms of CT must overcome the obstacle of proteolytic clearance. This clearance is especially rapid in the presence of gastrointestinal enzymes, with full degradation of both hCT and sCT occurring within a few hours. ${ }^{[158-161]}$ Experiments by Doschak and colleagues also revealed a $75 \%$ loss of radiolabeled sCT within 3 hours in major tissues in rat, with near complete loss within 24 hours. ${ }^{[162]}$ Indeed, the rapid degradation of CT particularly in the digestive track is a major obstacle to the development of oral formulations. Many prominent alternative oral delivery methods (detailed below) and peptide modifications are therefore focused on improving uptake and longevity. PEGylated and N-terminally acylated sCT have both shown improved resistance and intestinal permeability, with PEGylated sCT also showing increased longevity and a $\sim 3-$ $5 \times$ improvement in hypocalcemic effect in rat models. ${ }^{[161,163,164]}$ Lipidization has also been used to improve transepithelial transport, with both reversible and nonreversible additions being explored. ${ }^{[161,162]}$ Reversible lipidization of sCT maintain more peptide in serum for longer, with correspondingly longer hypocalcemic activity. ${ }^{[163]}$ Additionally, nonreversible lipidization has been shown to alter peptide secondary structure, which would likely inhibit receptor activation. ${ }^{[161]}$ As such, reversible lipidizations of $\mathrm{sCT}$ appear to be the more promising of the two for peptide modification.

\subsection{Alternative Delivery Methods}

Alternatives to injected and nasal spray sCT (the current preferred methods of administration) are being explored. The majority of these efforts focus on oral administration through novel delivery vessels. An oral mucoadhesive polymer delivery system developed by Gupta and colleagues demonstrated significant load delivery in rat intestine, and a 50-fold improvement in bioavailability versus direct intestinal injection. ${ }^{[164]}$ Biodegradable nanoparticles, coated liposomes, and lipid nanocapsules have all also been shown to produce improved sCT delivery, both in cultured cells and through oral delivery in vivo. ${ }^{[125,126,147,148]}$ Despite these advances, phase III clinical trials of oral delivery methods have failed to demonstrate statistically significant increases in bone-mineral density over nasal applications, and thus, the development of oral delivery methods for $\mathrm{SCT}$ remains in progress. ${ }^{[156,157,165]}$ The efficacy of all sCT treatment methods, including oral 
delivery, is likely still limited by sCT's aggregation and limited bioavailability upon absorption.

\subsection{CT Therapy and Medullary Thyroid Carcinoma}

A commonly mentioned concern regarding the use of CT in osteoporosis therapy is the correlation of CT fibrils with medullary thyroid carcinoma (MTC) tumor cells. ${ }^{[166-168]}$ It has been strongly suggested that CT fibrillation is a driver of tumor formation and progression. ${ }^{[30,112,132,146]}$ Several problems exist with this interpretation, however. MTC is a cancer of the CT-expressing Ccells, thus one would expect unmitigated cell proliferation to result in excessive local production of CT and subsequent fibril formation. To our knowledge, as of this writing, there is no evidence in the literature showing CT, fibrillar or otherwise, causes MTC, with this idea being further challenged by the existence of CT-negative MTC tumors. ${ }^{[169,170]}$ CT's use as a biomarker for MTC also suggests it to be an effect, rather than a cause, of MTC, since elevated CT serum levels occur after tumor formation. ${ }^{[171,172]}$ Additionally, elevated CT levels do not necessarily correspond to the presence of MTC. ${ }^{[173]}$ The strongest case for an active role in MTC for CT is an analysis by the Food and Drug Administration and the European Medicines Agency, finding CT therapy correlates with cancer risk. ${ }^{[174]}$ However, this correlation was weak $(<$ $1 \%$ increase), involved clinical trials much longer than current typical dosing regimens for short-term use, and showed no specificity to MTC versus other carcinomas. ${ }^{[174]}$ Overall, the fibrillation of CT seems like a consequence, rather than a driver, of MTC.

\section{Summary and Outlook}

In the 54 years since its discovery, the function of CT in the body, along with its methods of action, has been well characterized. Its roles in skeletal protection and hypercalcemia prevention have made it an attractive option as a therapeutic agent, and the last 15 years have seen remarkable progress in CT research, in both therapeutic and structural contexts. Peptide modifications and alternative delivery methods have increased the bioavailability and convenience of CT therapy, and through the use of NMR and other advanced methodologies, the structures of CT fibers have been illuminated. The effects of lipids on $\mathrm{CT}$ aggregation provides strong potential measures of control in therapeutic delivery and hints at possible physiological roles for their interactions. Additionally, studies focusing on the structure and function of early aggregation intermediates have yielded clues as to their role in aggregation, and have clarified key secondary structural motifs for both aggregation and biological activity. Using this information, CT mutants have been developed which hold promise as both therapeutics and tools to further understand CT-receptor interactions.

Despite these facts, significant questions still remain regarding CT. While it remains unlikely that CT will supplant more popular long-term osteoporosis treatment options, such as bisphosphonates, its utility in select circumstances merits continued work to improve its immune effects and limited bioavailability. Efforts to develop oral CT delivery systems hold particular promise for the future, with multiple possible candidates undergoing clinical trials. While early events in CT aggregation have received more attention recently, as with all amyloid peptides, further studies are required before a functional understanding of the aggregation intermediates can be constructed. In particular, an understanding of the role of the central $\alpha$-helix and $\mathrm{N}$-terminal loop of $\mathrm{CT}$ in mediating interactions with the CTR is required. Experiments yielding residue-specific information on the role of the C-terminus in receptor interactions preview an exciting avenue for further elucidation of the structure-function relationship of CT-CTR interactions. Such information would greatly aid in informing peptide modifications that decrease aggregation while maintaining or enhancing biological activity. Such CT mutants would prove invaluable in improving $\mathrm{CT}$ therapeutics, regardless of delivery mechanism. Finally, while this review has considered aggregation in the context of therapeutic applications and biological function, an understanding of the aggregation of CT, specifically the lag phase, would provide information significant to the broader field of amyloid aggregation, with relevance to a range of disease pathologies.

\section{References}

[1] D. H. Copp, E. C. Cameron, B. A. Cheney, A. G. Davidson, K. G. Henze, Endocrinology 1962, 70, 638-649.

[2] M. Ashwini Kumar, G. V. Foster, I. Macintyre, Lancet 1963, 282, 480-482.

[3] P. F. Hirsch, G. F. Gauthier, P. L. Munson, Endocrinology 1963, 73, 244-252.

[4] H. B. Brewer, R. Ronan, Proc. Natl. Acad. Sci. U.S.A. 1969, 63, 940-947.

[5] H. D. Niall, H. T. Keutmann, D. H. Copp, J. T. Potts, Proc. Natl. Acad. Sci. U.S.A. 1969, 64, 771-778.

[6] J. T. Potts, H. D. Niall, H. T. Keutmann, H. B. Brewer, L. J. Deftos, Proc. Natl. Acad. Sci. U.S.A. 1968, 59, 1321-1328.

[7] B. Riniker, M. Brugger, B. Kamber, W. Rittel, P. Sieber, R. Neher, Biochem. J. 1969, 111, 14P.

[8] M. Zaidi, A. Inzerillo, B. Moonga, P. J. Bevis, C. L.-H. Huang, Bone 2002, 30, 655-663.

[9] D. Raulais, J. Hagaman, D. A. Ontjes, R. L. Lundblad, H. S. Kingdon, Eur. J. Biochem. 1976, 64, 607-611.

[10] Y. Li, J. Yan, X. Zhang, K. Huang, Proteins Struct. Funct. Bioinf. 2013, 81, 1862-1873.

[11] T. Arvinte; A. Cudd, A. F. Drake, J. Biol. Chem. 1993, 268, $6415-6422$

[12] M. Diociaiuti, G. Macchia, S. Paradisi, C. Frank, S. Camerini, P. Chistolini, M. C. Gaudiano, T. C. Petrucci, F. Mal- 
chiodi-Albedi, Biochim. Biophys. Acta 2014, 1842, 16221629.

[13] D. L. Rymer, T. A. Good, J. Biol. Chem. 2001, 276, $2523-$ 2530.

[14] G. Siligardi, B. Samorí, S. Melandri, M. Visconti, A. F. Drake, Eur. J. Biochem. 1994, 221, 1117-1125.

[15] I. W. Hamley, Chem. Rev. 2012, 112, 5147-5192.

[16] R. S. Harrison, P. C. Sharpe, Y. Singh, D. P. Fairlie, Rev. Physiol. Biochem. Pharmacol. 2007, 159, 1-77.

[17] P. J. Gilchrist, J. P. Bradshaw, Biochim. Biophys. Acta 1993, 1182, 111-114.

[18] F. Chiti, C. M. Dobson, Annu. Rev. Biochem. 2006, 75, 333-366.

[19] M. G. Spillantini, M. L. Schmidt, V. M. Lee, J. Q. Trojanowski, R. Jakes, M. Goedert, Nature 1997, 388, 839-840.

[20] P. Westermark, A. Andersson, G. T. Westermark, Physiol. Rev. 2011, 91, 795-826.

[21] M. P. Murphy, H. LeVine, J. Alzheimer's Dis. 2010, 19, 311-323.

[22] S. B. Prusiner, Science 1982, 216, 136-144.

[23] A. S. DeToma, S. Salamekh, A. Ramamoorthy, M. H. Lim, Chem. Soc. Rev. 2012, 41, 608-621.

[24] J. R. Brender, S. Salamekh, A. Ramamoorthy, Acc. Chem. Res. 2012, 45, 454-462.

[25] T. P. J. Knowles, M. Vendruscolo, C. M. Dobson, Nat. Rev. Mol. Cell Biol. 2014, 15, 384-396.

[26] J. E. Straub, D. Thirumalai, Annu. Rev. Phys. Chem. 2011, 62, 437-463.

[27] H. R. Patel, A. S. Pithadia, J. R. Brender, C. A. Fierke, A. Ramamoorthy, J. Phys. Chem. Lett. 2014, 5, 1864-1870.

[28] S. A. Kotler, J. R. Brender, S. Vivekanandan, Y. Suzuki, K. Yamamoto, M. Monette, J. Krishnamoorthy, P. Walsh, M. Cauble, M. M. B. Holl, E. N. G. Marsh, A. Ramamoorthy, Sci. Rep. 2015, 5, 11811.

[29] G. Meisl, J. B. Kirkegaard, P. Arosio, T. C. T. Michaels, M. Vendruscolo, C. M. Dobson, S. Linse, T. P. J. Knowles, Nat. Protoc. 2016, 11, 252-272.

[30] D. Schubert, C. Behl, R. Lesley, A. Brack, R. Dargusch, Y. Sagara, H. Kimura, Proc. Natl. Acad. Sci. U.S.A. 1995, 92, 1989-1993.

[31] K. Morimoto, S. Nagata, T. Kubo, T. Oda, I. Kaneko, Nihon Yakurigaku Zasshi 1998, 112 Suppl., 83P-87P.

[32] M. Diociaiuti, L. Z. Polzi, L. Valvo, F. Malchiodi-Albedi, C. Bombelli, M. C. Gaudiano, Biophys. J. 2006, 91, 22752281.

[33] D. Meleleo, V. Picciarelli, Biometals 2016, 29, 61-79.

[34] J. C. Robin, J. L. Ambrus, Res. Commun. Chem. Pathol. Pharmacol. 1982, 35, 491-498.

[35] C. H. Chesnut, M. Azria, S. Silverman, M. Engelhardt, M. Olson, L. Mindeholm, Osteoporosis Int. 2008, 19, 479-491.

[36] A. Cudd, T. Arvinte, R. E. Gaines Das, C. Chinni, I. MacIntyre, J. Pharm. Sci. 1995, 84, 717-719.

[37] F. R. Singer, R. S. Fredericks, C. Minkin, Arthritis Rheum. 1980, 23, 1148-1154.

[38] F. R. Singer, J. P. Aldred, R. M. Neer, S. M. Krane, J. T. Potts, K. J. Bloch, J. Clin. Invest. 1972, 51, 2331-2338.

[39] G. Bussolati, A. G. Pearse, J. Endocrinol. 1967, 37, $205-$ 209.

[40] L. Masi, M. L. Brandi, Clin. Cases Miner. Bone Metab. 2007, 4, 117-122.

[41] S. J. Wimalawansa, Crit. Rev. Neurobiol. 1997, 11, 167-239.

[42] P. F. Hirsch, H. Baruch, Endocrine 2003, 21, 201-208.

[43] G. V. Foster, Postgrad. Med. J. 1968, 44, 411-422.

[44] J. Friedman, L. G. Raisz, Science 1965, 150, 1465-1467.
[45] S. Wallach, G. Rousseau, L. Martin, M. Azria, Bone 1999, $25,509-516$.

[46] G. C. Nicholson, J. M. Moseley, P. M. Sexton, F. A. Mendelsohn, T. J. Martin, J. Clin. Invest. 1986, 78, 355-360.

[47] M. E. Holtrop, L. G. Raisz, H. A. Simmons, J. Cell Biol. 1974, 60, 346-355.

[48] T. J. Chambers, C. J. Magnus, J. Pathol. 1982, 136, 27-39.

[49] T. J. Chambers, K. Fuller, J. A. Darby, J. Cell. Physiol. 1987, 132, 90-96.

[50] S.-M. Lee, D. L. Hay, A. A. Pioszak, J. Biol. Chem. 2016, $291,8686-8700$.

[51] D. L. Hay, C. S. Walker, J. J. Gingell, G. Ladds, C. A. Reynolds, D. R. Poyner, Biochem. Soc. Trans. 2016, 44, 568573.

[52] Z. Zhang, L. Neff, A. L. M. Bothwell, R. Baron, W. C. Horne, Bone 2002, 31, 359-365.

[53] J.-F. Shyu, C. Shih, C.-Y. Tseng, C.-H. Lin, D.-T. Sun, H.-T. Liu, H.-C. Tsung, T.-H. Chen, R.-B. Lu, Bone 2007, 40, $1329-1342$.

[54] F. Malchiodi-Albedi, V. Contrusciere, C. Raggi, K. Fecchi, G. Rainaldi, S. Paradisi, A. Matteucci, M. T. Santini, M. Sargiacomo, C. Frank, M. C. Gaudiano, M. Diociaiuti, Biochim. Biophys. Acta 2010, 1802, 406-415.

[55] V. Stipani, E. Gallucci, S. Micelli, V. Picciarelli, R. Benz, Biophys. J. 2001, 81, 3332-3338.

[56] H. Kajiya, Adv. Exp. Med. Biol. 2012, 740, 917-932.

[57] X. Cheng, E. S. Hookway, T. Kashima, U. Oppermann, A. Galione, N. A. Athanasou, Calcif. Tissue Int. 2015, 96, $73-$ 79.

[58] Y. Okubo, K. Bessho, K. Fujimura, K. Kusumoto, Y. Ogawa, T. Iizuka, Biochem. Biophys. Res. Commun. 2000, 269, 317-321.

[59] M. C. Monier-Faugere, Z. Geng, Q. Qi, I. Arnala, H. H. Malluche, J. Bone Miner. Res. 1996, 11, 446-455.

[60] L. I. Plotkin, R. S. Weinstein, A. M. Parfitt, P. K. Roberson, S. C. Manolagas, T. Bellido, J. Clin. Invest. 1999, 104, $1363-1374$.

[61] J. H. Gooi, S. Pompolo, M. A. Karsdal, N. H. Kulkarni, I. Kalajzic, S. H. M. McAhren, B. Han, J. E. Onyia, P. W. M. Ho, M. T. Gillespie, N. C. Walsh, L. Y. Chia, J. M. W. Quinn, T. J. Martin, N. A. Sims, Bone 2010, 46, 1486-1497.

[62] R. A. Davey, D. M. Findlay, J. Bone Miner. Res. 2013, 28, 973-979.

[63] D. L. Hurley, R. D. Tiegs, H. W. Wahner, H. Heath, N. Engl. J. Med. 1987, 317, 537-541.

[64] A. O. Hoff, P. Catala-Lehnen, P. M. Thomas, M. Priemel, J. M. Rueger, I. Nasonkin, A. Bradley, M. R. Hughes, N. Ordonez, G. J. Cote, M. Amling, R. F. Gagel, J. Clin. Invest. 2002, 110, 1849-1857.

[65] A. K. Huebner, J. Keller, P. Catala-Lehnen, S. Perkovic, T. Streichert, R. B. Emeson, M. Amling, T. Schinke, Arch. Biochem. Biophys. 2008, 473, 210-217.

[66] A. K. Huebner, T. Schinke, M. Priemel, S. Schilling, A. F. Schilling, R. B. Emeson, J. M. Rueger, M. Amling, J. Bone Miner. Res. 2006, 21, 1924-1934.

[67] S. B. Baylin, A. L. Bailey, T.-H. Hsu, G. V. Foster, Metabolism 1977, 26, 1345-1354.

[68] J. N. M. Heersche, Bone Miner. 1992, 16, 174-177.

[69] R. A. Davey, A. G. Turner, J. F. McManus, W. S. M. Chiu, F. Tjahyono, A. J. Moore, G. J. Atkins, P. H. Anderson, C. Ma, V. Glatt, H. E. MacLean, C. Vincent, M. Bouxsein, H. A. Morris, D. M. Findlay, J. D. Zajac, J. Bone Miner. Res. 2008, 23, 1182-1193. 
[70] J. C. Stevenson, C. J. Hillyard, I. MacIntyre, H. Cooper, M. I. Whitehead, Lancet 1979, 2, 769-770.

[71] R. Kumar, W. R. Cohen, P. Silva, F. H. Epstein, J. Clin. Invest. 1979, 63, 342-344.

[72] J. P. Woodrow, C. J. Sharpe, N. J. Fudge, A. O. Hoff, R. F. Gagel, C. S. Kovacs, Endocrinology 2006, 147, 4010-4021.

[73] P. Jaeger, W. Jones, T. L. Clemens, J. P. Hayslett, J. Clin. Invest. 1986, 78, 456-461.

[74] L. G. Raisz, C. L. Trummel, M. F. Holick, H. F. DeLuca, Science 1972, 175, 768-769.

[75] A. J. Felsenfeld, B. S. Levine, Clin. Kidney J. 2015, 8, 180 187.

[76] T. J. Martin, R. A. Melick, Australas. Ann. Med. 1969, 18, $258-263$.

[77] C. W. Cooper, P. F. Hirsch, S. U. Toverud, P. L. Munson, Endocrinology 1967, 81, 610-616.

[78] P. F. Hirsch, P. L. Munson, Physiol. Rev. 1969, 49, 548-622.

[79] A. M. Parfitt, Bone 2003, 33, 259-263.

[80] G. J. Atkins, D. M. Findlay, Osteoporosis Int. 2012, 23, 2067-2079.

[81] E. M. Brown, Best Pract. Res. Clin. Endocrinol. Metab. 2013, 27, 333-343.

[82] H. Ohno, M. Noguchi, N. Takayanagi, Jpn. J. Pharmacol. 1985, 37, 67-75.

[83] C. W. Cooper, W. H. Schwesinger, A. M. Mahgoub, D. A. Ontjes, Science 1971, 172, 1238-1240.

[84] G. S. Cottrell, F. Alemi, J. G. Kirkland, E. F. Grady, C. U. Corvera, A. Bhargava, Peptides 2012, 35, 202-211.

[85] A. D. Care, J. B. Bruce, J. Boelkins, A. D. Kenny, H. Conaway, C. S. Anast, Endocrinology 1971, 89, 262-271.

[86] K. Kanaori, A. Y. Nosaka, Biochemistry 1995, 34, $12138-$ 12143.

[87] D. F. Moriarty, S. Vagts, D. P. Raleigh, Biochem. Biophys. Res. Commun. 1998, 245, 344-348.

[88] C. Avidan-Shpalter, E. Gazit, Amyloid 2006, 13, 216-225.

[89] S. Micelli, D. Meleleo, V. Picciarelli, M. G. Stoico, E. Gallucci, Biophys. J. 2004, 87, 1065-1075.

[90] J. R. Brender, J. Krishnamoorthy, M. F. M. Sciacca, S. Vivekanandan, L. D'Urso, J. Chen, C. La Rosa, A. Ramamoorthy, J. Phys. Chem. B 2015, 119, 2886-2896.

[91] L. Hong, X. Qi, Y. Zhang, J. Phys. Chem. B 2012, 116, 6611-6617.

[92] P. Arosio, T. P. J. Knowles, S. Linse, Phys. Chem. Chem. Phys. 2015, 17, 7606-7618.

[93] H. Itoh-Watanabe, M. Kamihira-Ishijima, I. Kawamura, M. Kondoh, M. Nakakoshi, M. Sato, A. Naito, Phys. Chem. Chem. Phys. 2013, 15, 16956-16964.

[94] M. C. Gaudiano, M. Colone, C. Bombelli, P. Chistolini, L. Valvo, M. Diociaiuti, Biochim. Biophys. Acta 2005, 1750, $134-145$.

[95] M. C. Gaudiano, M. Diociaiuti, P. Bertocchi, L. Valvo, Biochim. Biophys. Acta 2003, 1623, 33-40.

[96] N. Rastogi, K. Mitra, D. Kumar, R. Roy, Inorg. Chem. 2012, 51, 5642-5650.

[97] M. F. M. Sciacca, S. A. Kotler, J. R. Brender, J. Chen, D. Lee, A. Ramamoorthy, Biophys. J. 2012, 103, 702-710.

[98] M. Kamihira, A. Naito, S. Tuzi, A. Y. Nosaka, H. Saitô, Protein Sci. 2000, 9, 867-877.

[99] A. Naito, M. Kamihira, R. Inoue, H. Saitô, Magn. Reson. Chem. 2004, 42, 247-257.

[100] R. Huang, S. Vivekanandan, J. R. Brender, Y. Abe, A. Naito, A. Ramamoorthy, J. Mol. Biol. 2012, 416, 108-120.

[101] M. Reches, Y. Porat, E. Gazit, J. Biol. Chem. 2002, 277, $35475-35480$
[102] D. Zanuy, N. Haspel, H.-H. G. Tsai, B. Ma, K. Gunasekaran, H. J. Wolfson, R. Nussinov, Phys. Biol. 2004, 1, 89-99.

[103] A. Lakshmanan, D. W. Cheong, A. Accardo, E. Di Fabrizio, C. Riekel, C. A. E. Hauser, Proc. Natl. Acad. Sci. U.S.A. 2013, 110, 519-524.

[104] A. Rawat, D. Kumar, J. Pept. Sci. 2013, 19, 33-45.

[105] M. Kamihira, Y. Oshiro, S. Tuzi, A. Y. Nosaka, H. Saitô, A. Naito, J. Biol. Chem. 2003, 278, 2859-2865.

[106] H. Itoh-Watanabe, M. Kamihira-Ishijima, N. Javkhlantugs, R. Inoue, Y. Itoh, H. Endo, S. Tuzi, H. Saitô, K. Ueda, A. Naito, Phys. Chem. Chem. Phys. 2013, 15, 8890-8901.

[107] M. Guo, P. M. Gorman, M. Rico, A. Chakrabartty, D. V. Laurents, FEBS Lett. 2005, 579, 3574-3578.

[108] K. E. Marshall, K. L. Morris, D. Charlton, N. O'Reilly, L. Lewis, H. Walden, L. C. Serpell, Biochemistry 2011, 50, $2061-2071$.

[109] M. Diociaiuti, M. C. Gaudiano, F. Malchiodi-Albedi, Int. J. Mol. Sci. 2011, 12, 9277-9295.

[110] E. Gazit, FASEB J. 2002, 16, 77-83.

[111] A. Bertolani, L. Pirrie, L. Stefan, N. Houbenov, J. S. Haataja, L. Catalano, G. Terraneo, G. Giancane, L. Valli, R. Milani, O. Ikkala, G. Resnati, P. Metrangolo, Nat. Commun. 2015, 6, 7574.

[112] H.-H. Tsai, D. Zanuy, N. Haspel, K. Gunasekaran, B. Ma, C.-J. Tsai, R. Nussinov, Biophys. J. 2004, 87, 146-158.

[113] Protein misfolding diseases: current and emerging principles and therapies (Eds.: M. Ramirez-Alvarado, J. W. Kelly, C. M. Dobson), John Wiley \& Sons, Inc., Hoboken, 2010.

[114] A. Shtainfeld, T. Sheynis, R. Jelinek, Biochemistry 2010, $49,5299-5307$.

[115] V. A. Iconomidou, A. Leontis, A. Hoenger, S. J. Hamodrakas, FEBS Lett. 2013, 587, 569-574.

[116] K. Wagner, N. Van Mau, S. Boichot, A. V. Kajava, U. Krauss, C. Le Grimellec, A. Beck-Sickinger, F. Heitz, Biophys. J. 2004, 87, 386-395.

[117] H. H. Bauer, M. Müller, J. Goette, H. P. Merkle, U. P. Fringeli, Biochemistry 1994, 33, 12276-12282.

[118] T. Arvinte, A. F. Drake, J. Biol. Chem. 1993, 268, 64086414.

[119] J. P. Meyer, J. T. Pelton, J. Hoflack, V. Saudek, Biopolymers 1991, 31, 233-241.

[120] S. Micelli, D. Meleleo, V. Picciarelli, E. Gallucci, Front. Biosci. 2006, 11, 2035-2044.

[121] S. B. Fowler, S. Poon, R. Muff, F. Chiti, C. M. Dobson, J. Zurdo, Proc. Natl. Acad. Sci. U.S.A. 2005, 102, $10105-$ 10110.

[122] S. D. Stroop, H. Nakamuta, R. E. Kuestner, E. E. Moore, R. M. Epand, Endocrinology 1996, 137, 4752-4756.

[123] H. Kawashima, M. Katayama, R. Yoshida, K. Akaji, A. Asano, M. Doi, J. Pept. Sci. 2016, 22, 480-484.

[124] A. M. Morris, M. A. Watzky, J. N. Agar, R. G. Finke, Biochemistry 2008, 47, 2413-2427.

[125] A. Umerska, N. Matougui, A.-C. Groo, P. Saulnier, Int. J. Pharm. 2016, 506, 191-200.

[126] N. Matougui, L. Boge, A.-C. Groo, A. Umerska, L. Ringstad, H. Bysell, P. Saulnier, Int. J. Pharm. 2016, 502, 80-97.

[127] L. Baginski, O. L. Gobbo, F. Tewes, J. J. Salomon, A. M. Healy, U. Bakowsky, C. Ehrhardt, Pharm. Res. 2012, 29, $1425-1434$.

[128] T. Sheynis, R. Jelinek, J. Phys. Chem. B 2010, 114, 1553015535.

[129] F. Malchiodi-Albedi, S. Paradisi, A. Matteucci, C. Frank, M. Diociaiuti, Int. J. Alzheimer's Dis. 2011, 2011, 906964. 
[130] A. Motta, G. Andreotti, P. Amodeo, G. Strazzullo, M. A. Castiglione Morelli, Proteins 1998, 32, 314-323.

[131] R. M. Epand, R. F. Epand, R. C. Orlowski, R. J. Schlueter, L. T. Boni, S. W. Hui, Biochemistry 1983, 22, 5074-5084.

[132] S. S.-S. Wang, T. A. Good, D. L. Rymer, Protein Sci. 2005, 14, 1419-1428.

[133] R. Sabaté, J. Estelrich, J. Phys. Chem. B 2005, 109, 1102711032.

[134] P. Amodeo, A. Motta, G. Strazzullo, M. A. Castiglione Morelli, J. Biomol. NMR 1999, 13, 161-174.

[135] G. Andreotti, B. L. Méndez, P. Amodeo, M. A. C. Morelli, H. Nakamuta, A. Motta, J. Biol. Chem. 2006, 281, $24193-$ 24203.

[136] E. Johansson, J. L. Hansen, A. M. K. Hansen, A. C. Shaw, P. Becker, L. Schäffer, S. Reedtz-Runge, J. Biol. Chem. 2016, 291, 13689-13698.

[137] G. Andreotti, R. M. Vitale, C. Avidan-Shpalter, P. Amodeo, E. Gazit, A. Motta, J. Biol. Chem. 2011, 286, $2707-2718$

[138] D. Schneider, M. T. Hofmann, J. A. Peterson, Am. Fam. Physician 2002, 65, 2069-2072.

[139] K. Henriksen, A.-C. Bay-Jensen, C. Christiansen, M. A. Karsdal, Expert Opin. Biol. Ther. 2010, 10, 1617-1629.

[140] C. Feletti, V. Bonomini, Nephron 1979, 24, 85-88.

[141] Y. Yamamoto, H. Nakamuta, M. Koida, J. K. Seyler, R. C. Orlowski, Jpn. J. Pharmacol. 1982, 32, 1013-1017.

[142] F. Levy, R. Muff, S. Dotti-Sigrist, M. A. Dambacher, J. A Fischer, J. Clin. Endocrinol. Metab. 1988, 67, 541-545.

[143] A. Grauer, H. H. Reinel, R. Ziegler, F. Raue, Horm. Metab. Res. 1993, 25, 486-488.

[144] R. Maier, B. Kamber, B. Riniker, W. Rittel, Clin. Endocrinol. 1976, 5, 327S-332S.

[145] G. Andreotti, A. Motta, J. Biol. Chem. 2003, 279, 63646370.

[146] C. Guo, L. Ma, Y. Zhao, A. Peng, B. Cheng, Q. Zhou, L. Zheng, K. Huang, Sci. Rep. 2015, 5, 13556.

[147] H. S. Yoo, T. G. Park, J. Pharm. Sci. 2004, 93, 488-495.

[148] M. Werle, H. Takeuchi, Int. J. Pharm. 2009, 370, 26-32.

[149] J. Zurdo, Protein Pept. Lett. 2005, 12, 171-187.

[150] Y. Kallberg, M. Gustafsson, B. Persson, J. Thyberg, J. Johansson, J. Biol. Chem. 2001, 276, 12945-12950.

[151] V. Villegas, J. Zurdo, V. V. Filimonov, F. X. Avilés, C. M. Dobson, L. Serrano, Protein Sci. 2000, 9, 1700-1708.

[152] S. H. Tella, J. C. Gallagher, J. Steroid Biochem. Mol. Biol. 2014, 142, 155-170.

[153] A. Tagiyev, H. Demirbilek, B. Tavil, G. Buyukyilmaz, F. Gumruk, M. Cetin, J. Pediatr. Hematol. Oncol. 2016, 38, $232-234$.
[154] M. A. Abbassy, I. Watari, A. S. Bakry, T. Ono, A. H. Hassan, Int. J. Oral Sci. 2016, 8, 39-44.

[155] J. Wei, J. Wang, Y. Gong, R. Zeng, Mol. Med. Rep. 2015, $12,1717-1726$.

[156] N. Binkley, M. Bolognese, A. Sidorowicz-Bialynicka, T. Vally, R. Trout, C. Miller, C. E. Buben, J. P. Gilligan, D. S. Krause, J. Bone Miner. Res. 2012, 27, 1821-1829.

[157] L. Bandeira, E. M. Lewiecki, J. P. Bilezikian, Expert Opin. Drug Metab. Toxicol. 2016, 12, 1-9.

[158] Y. S. Youn, J. Y. Jung, S. H. Oh, S. D. Yoo, K. C. Lee, J. Controlled Release 2006, 114, 334-342.

[159] A. Mero, M. Schiavon, F. M. Veronese, G. Pasut, J. Controlled Release 2011, 154, 27-34.

[160] S. Trier, L. Linderoth, S. Bjerregaard, H. M. Strauss, U. L. Rahbek, T. L. Andresen, Eur. J. Pharm. Biopharm. 2015, 96, 329-337.

[161] W. Cheng, S. Satyanarayanajois, L.-Y. Lim, Pharm. Res. 2007, 24, 99-110.

[162] J. Wang, D. Chow, H. Heiati, W.-C. Shen, J. Controlled Release 2003, 88, 369-380.

[163] W. Cheng, L.-Y. Lim, Mol. Pharm. 2008, 5, 610-621.

[164] V. Gupta, B. H. Hwang, J. Lee, A. C. Anselmo, N. Doshi, S. Mitragotri, J. Controlled Release 2013, 172, 753-762.

[165] P. Varamini, I. Toth, Expert Opin. Drug Delivery 2016, 13, 507-522.

[166] R. Khurana, A. Agarwal, V. K. Bajpai, N. Verma, A. K. Sharma, R. P. Gupta, K. P. Madhusudan, Endocrinology 2004, 145, 5465-5470.

[167] M. Tubiana, G. Milhaud, G. Coutris, J. Lacour, C. Parmentier, B. Bok, Br. Med. J. 1968, 4, 87-89.

[168] K. Sletten, P. Westermark, J. B. Natvig, J. Exp. Med. 1976, 143, 993-998.

[169] K. W. Schmid, C. Ensinger, Virchows Arch. 1998, 433, 209215.

[170] M. T. Samà, R. Rossetto Giaccherino, M. Gallo, F. Felicetti, F. Maletta, N. Bonelli, A. Piovesan, N. Palestini, E. Ghigo, E. Arvat, J. Cancer Res. Clin. Oncol. 2016, 142, 2023-2029.

[171] Y. Ito, A. Miyauchi, M. Kihara, T. Kudo, A. Miya, Endocr. J. 2016, 63, 663-667.

[172] Y. J. Bae, M. Schaab, J. Kratzsch, Recent Results Cancer Res. 2015, 204, 117-137.

[173] P. W. Rosario, M. R. Calsolari, Horm. Metab. Res. 2016, 48, $372-276$.

[174] R. A. Overman, M. Borse, M. L. Gourlay, Ann. Pharmacother. 2013, 47, 1675-1684.

Received: July 22, 2016 Published online: November 15, 2016 